\title{
Alternative proofs of linear response for piecewise expanding unimodal maps
}

\author{
VIVIANE BALADI $\dagger$ and DANIEL SMANIA $\ddagger$ \\ $\dagger$ D.M.A., UMR 8553, École Normale Supérieure, 75005 Paris, France \\ (e-mail: viviane.baladi@ens.fr) \\ $\ddagger$ Departamento de Matemática, ICMC-USP, Caixa Postal 668, São Carlos-SP, \\ CEP 13560-970, São Carlos-SP, Brazil \\ (e-mail:smania@icmc.usp.br)
}

(Received 9 April 2008 and accepted in revised form 17 November 2008)

\begin{abstract}
We give two new proofs that the Sinai-Ruelle-Bowen (SRB) measure $t \mapsto \mu_{t}$ of a $C^{2}$ path $f_{t}$ of unimodal piecewise expanding $C^{3}$ maps is differentiable at 0 if $f_{t}$ is tangent to the topological class of $f_{0}$. The arguments are more conceptual than the original proof of Baladi and Smania [Linear response formula for piecewise expanding unimodal maps. Nonlinearity 21 (2008), 677-711], but require proving Hölder continuity of the infinitesimal conjugacy $\alpha$ (a new result, of independent interest) and using spaces of bounded $p$-variation. The first new proof gives differentiability of higher order of $\int \psi d \mu_{t}$ if $f_{t}$ is smooth enough and stays in the topological class of $f_{0}$ and if $\psi$ is smooth enough (a new result). In addition, this proof does not require any information on the decomposition of the SRB measure into regular and singular terms, making it potentially amenable to extensions to higher dimensions. The second new proof allows us to recover the linear response formula (i.e. the formula for the derivative at 0) obtained by Baladi and Smania, by an argument more conceptual than the 'brute force' cancellation mechanism used by Baladi and Smania.
\end{abstract}

\section{Introduction}

Many chaotic dynamical systems $f: M \rightarrow M$ on a Riemannian manifold $M$ admit a SinaiRuelle-Bowen (SRB) measure $\mu$ (see e.g. [23]) which describes the statistical properties of a 'large' set of initial conditions in the sense of Lebesgue measure. (In dimension one, a SRB measure is simply an absolutely continuous ergodic invariant probability measure $\mu_{t}=\rho_{t} d x$, with a positive Lyapunov exponent.) It is of interest (in particular, in view of applications to statistical mechanics, see e.g. $[\mathbf{1 8}, \mathbf{2 0}]$ ) to study the smoothness of $t \mapsto \mu_{t}$, when $f_{t}$ is a smooth family of dynamical systems, each having a SRB measure $\mu_{t}$. If $t \mapsto \mu_{t}$ is differentiable, we say that the linear response holds. Ruelle [18] obtained not 
only differentiability, but also a formula for the derivative (the linear response formula), in the case of smooth uniformly hyperbolic dynamical systems. (See [19] for the formulas for higher-order derivatives, without proofs. Differentiability of higher order in this framework was subsequently proved in $[\mathbf{9 , 1 0}]$, using 'modern' Banach spaces.)

In [4], we proved that the SRB measure $t \mapsto \mu_{t}$ of a $C^{2}$ family of piecewise $C^{3}$ and piecewise expanding unimodal maps $f_{t}$, with $f_{0}$ mixing (see $\$ 2.1$ for formal definitions), is differentiable at $t=0$ (as a Radon measure) if and only if $f_{t}$ is tangent to the topological class of $f_{0}$ at $t=0$ (for the necessity of the tangency condition, we require an additional mild technical condition). Keller [14] proved a long time ago that $\rho_{t}$ has a $|t||\ln | t||$ modulus of continuity, as an element of $L^{1}(d x)$, and examples in $[\mathbf{1}, \mathbf{4}]$ show that this can be attained as a lower bound for non-tangential families. We also obtained in [4] a linear response formula analogous to that in [18], using a resummation introduced in [1].

More recently, differentiability of the SRB measure (in the weak sense, that is, as an appropriate distribution) was obtained $[6,21]$ for smooth families $f_{t}$ of analytic and nonuniformly expanding unimodal maps which stay in the topological class of $f_{0}$. The cases of families of smooth non-uniformly expanding interval maps only tangent to the topological class (where Whitney differentiability is expected on suitable subsets of parameters), as well as higher-dimensional dynamical systems such as piecewise expanding/hyperbolic maps or Hénon-like maps, are still open, and much more difficult, see [2] for a discussion. In particular, the arguments in [4] and [21] used detailed information about the structure of the SRB measure, decomposing it into a regular and a singular term. This type of information may be far less accessible in higher dimensions.

In this article, we give two new proofs of the fact [4, Theorem 5.1] that the SRB measure of a $C^{2}$ family of piecewise $C^{3}$ and piecewise expanding unimodal maps $f_{t}$, with $f_{0}$ mixing, is differentiable at $t=0$ if $f_{t}$ is tangent to the topological class of $f_{0}$ at $t=0$.

Section 3 contains our first new proof (see Corollary 3.2), more precisely, we obtain differentiability of $t \mapsto \int \psi d \mu_{t}$ for $\psi \in C^{1+\text { Lip }}$ if $f_{t}$ is a $C^{2}$ family of piecewise expanding $C^{3}$ unimodal maps tangent to the topological class of a mixing map $f_{0}$. The argument is based on thermodynamic formalism, using potentials $(s, t) \mapsto s\left(\psi \circ h_{t}\right)-$ $\log \left|f_{t}^{\prime} \circ h_{t}\right|$ (where $h_{t}$ conjugates $\tilde{f}_{t}$ with $f_{0}$ and $\left|\tilde{f}_{t}-f_{t}\right|=O\left(t^{2}\right)$ ) and does not require any knowledge about the structure of $\mu_{t}$. (Arguments for weak differentiability of Gibbs measures via thermodynamic formalism have been used previously in $[\mathbf{1 2}, \mathbf{1 3}]$, see $[\mathbf{1 3}$, Corollary 1, p 595].) This argument may therefore be useful in more difficult situations (such as Hénon maps, see [2]). It requires the Hölder differentiability of the infinitesimal conjugacy $\alpha$ to be proved, a new result (Proposition 2.3) of independent interest. Also, this new proof gives that $t \mapsto \int \psi d \mu_{t}$ is a $C^{j}$ function, if $\psi \in C^{j+\text { Lip }}$ and $f_{t}$ is a $C^{j+1}$ family of piecewise expanding $C^{j+2}$ maps in the topological class of $f_{0}$, for any $j \geq 1$ (this is a new result, Theorem 3.1). Note also that we do not require the assumption from [4] that there is a function $X$ so that $\left.\partial f_{t}\right|_{t=0}=X \circ f_{0}$.

The first new proof requires $\psi \in C^{1+\text { Lip }}$ (instead of $\psi \in C^{0}$ as in [4]) and does not furnish the linear response formula. Section 4 contains our second new proof (Theorem 4.1), which uses spectral perturbation theory for transfer operators associated with the dynamics $f_{0}$ and the weight $1 /\left|f_{t}^{\prime} \circ h_{t}\right|$. This other proof gives differentiability of $\int \psi d \mu_{t}$ for $\psi \in C^{0}$ and, using the assumption that $\left.\partial f_{t}\right|_{t=0}=X \circ f_{0}$, allows us to recover 
the linear response formula from [4]. (This second proof also uses the Hölder regularity of $\alpha$ from Proposition 2.3.) Note, however, that this second proof requires information on the structure of $\mu_{t}$ from [1, Proposition 3.3].

Putting together Theorems 3.1 and 4.1 (or Theorem 3.1 and [4, Theorem 5.1]), we obtain the following additional result (Corollary 4.4): if $f_{t}$ is a $C^{2}$ family of piecewise expanding $C^{3}$ unimodal maps in the topological class of $f_{0}$, then $t \mapsto \mu_{t}$ is $C^{1}$ from a neighbourhood of zero to Radon measures.

We emphasize that neither new proof proves that the condition to be tangent to the topological class is necessary, in contrast to the argument in [4] (see Theorem 7.1 there). The proofs here are a bit shorter than that given in [4], although the present account requires some results from our previous papers (such as [1, Proposition 3.3], [5, Proposition 3.2 and Theorem 2], [4, Proposition 2.4, Lemma 2.6 and Proposition 3.3]).

\section{Definitions and notation: Hölder smoothness of the infinitesimal conjugacy}

2.1. Formal definitions. We denote $I=[-1,1]$. For an integer $k \geq 1$, we define $\mathcal{B}^{k}$ to be the linear space of continuous functions $f: I \rightarrow \mathbb{R}$ such that $f$ is $C^{k}$ on the intervals $[-1,0]$ and $[0,1]$. Then $\mathcal{B}^{k}$ is a Banach space for the norm $\max \left\{|f|_{C^{k}([-1,0])},|f|_{C^{k}([0,1])}\right\}$. For an integer $k \geq 1$, we define the set $\mathcal{U}^{k}$ of piecewise expanding $C^{k}$ unimodal maps to be the set of $f \in \mathcal{B}^{k}$ such that $\dagger f(-1)=f(1)=-1$, inf $_{x \neq 0}\left|f^{\prime}(x)\right|>1$, and $f(0) \leq 1$ (so that $f(I) \subset I)$. The point $c=0$ is called the critical point of $f$.

A piecewise expanding $C^{k}$ unimodal map $f$ is good if either $c$ is not periodic under $f$ or $\left|\left(f^{q-1}\right)^{\prime}(f(c))\right| \min \left\{\left|f_{+}^{\prime}(c)\right|,\left|f_{-}^{\prime}(c)\right|\right\}>2$, where $q \geq 2$ is the minimal period of $c$; it is mixing if $f$ is topologically mixing on $\left[c_{2}, c_{1}\right]$, where $c_{k}=f^{k}(c)$.

For $1 \leq j \leq k$, a $C^{j}$ family of piecewise expanding $C^{k}$ unimodal maps is a $C^{j}$ map $f_{t}$ from $t \in(-\epsilon, \epsilon)$ to $\mathcal{U}^{k}$ for some $\epsilon>0$. (In this paper, $k \geq 1$ is an integer and $j$ is either an integer or $j=k-1+$ Lip for $k \geq 2$, the notation $\mathcal{B}^{k+\text { Lip }}$ and $\mathcal{U}^{k+\text { Lip }}$ for integers $k \geq 1$ being self-explanatory. See also Remark 2.2.)

Remark 2.1. A $C^{j}$ family $f_{t}$ of piecewise expanding $C^{k}$ unimodal maps is a $C^{j, k}$ perturbation of $f_{0}$ in the sense of [4] if $j=k \geq 2$.

Remark 2.2. Considering $\mathcal{B}^{k+\beta}$ and $\mathcal{U}^{k+\beta}$ for $k \geq 1$ integer and a Hölder exponent $0<\beta$ $<1$ will perhaps allow us to avoid the loss of regularity from $C^{k+1}$ to $C^{k+\mathrm{Lip}}$, e.g. in [4, Proposition 2.4] (this question was asked by J.-C. Yoccoz). However, since the spectral result of Wong [22] only holds on the space $B V_{p}$ of functions of bounded $p$-variation if $1 \leq p<p_{0}$, for some $p_{0}>1$ depending on the dynamics, it may be necessary in this case to replace $B V_{p}$ by spaces of generalized $p$-variation, as introduced by Keller [15]. (See also Remark 2.5.)

Assume that $f_{t}$ is a $C^{j}$ family of piecewise expanding $C^{k}$ unimodal maps for $k \geq j>1$. By classical results of Lasota-Yorke, each $f_{t}$ has a unique absolutely continuous invariant probability measure $\mu_{t}=\rho_{t} d x$. This measure is ergodic and it is called the SRB measure of $f_{t}$. If $f_{t}$ is mixing, then $\mu_{t}$ is mixing. If $f_{0}$ is good and mixing, then $f_{t}$ is mixing for all small enough $t$ (see [14] and references therein).

$\dagger$ A prime denotes differentiation with respect to $x \in I$, a priori in the sense of distributions. 
We say that a piecewise expanding $C^{k}$ unimodal map $g$ is in the topological class of $f$ if there is a homeomorphism $h: I \rightarrow I$ conjugating $f$ and $g$, that is $h \circ f=g \circ h$. This implies that $h(c)=c$. We say that a $C^{j}$ family $f_{t}$ of piecewise expanding $C^{k}$ unimodal maps is in the topological class of $f_{0}$ if there exist homeomorphisms $h_{t}: I \rightarrow I$ such that

$$
h_{t} \circ f_{0}=f_{t} \circ h_{t} \quad \text { for all }|t|<\epsilon .
$$

(This implies that $h_{t}(c)=c$ for all $c$.) We proved in [4, Proposition 2.4] that $(x, t) \mapsto h_{t}(x)$ is continuous, and that for each $x$ the map $t \mapsto h_{t}(x)$ is $C^{k+\mathrm{Lip}-1}$. Differentiability of $t \mapsto h_{t}(x)$ will play an important role in our arguments below. We say that a $C^{j}$ family $f_{t}$ of piecewise expanding $C^{k}$ unimodal maps $(k \geq j \geq 2)$ is tangent to the topological class of $f_{0}$ if there exists a $C^{j}$ family $\tilde{f}_{t}$ of piecewise expanding $C^{k}$ unimodal maps in the topological class of $f_{0}$ so that $\tilde{f}_{t}=f_{0}$ and $\left.\partial_{t} f_{t}\right|_{t=0}=\left.\partial_{t} \tilde{f}_{t}\right|_{t=0}$. (Note that there is a typographical mistake in $[4$, p. 682 , line 6$]$, where ' $C^{2,2}$ perturbation' should be replaced by ' $C^{r_{0}, r}$ perturbation'.)

We say that a bounded function $v: I \rightarrow \mathbb{R}$ is horizontal for $f$, if $v(-1)=v(1)=0$, and setting $M_{f}=q$ if $c$ is periodic of minimal period $q$, and $M_{f}=+\infty$ otherwise,

$$
J(f, v)=\sum_{j=0}^{M_{f}-1} \frac{v\left(c_{j}\right)}{\left(f^{j}\right)^{\prime}\left(c_{1}\right)}=0 .
$$

In [4, Corollary 2.6] we proved that if $f_{t}$ is a $C^{2}$ family of piecewise expanding $C^{2}$ unimodal maps tangent to the topological class of $f_{0}$, then $v=\left.\partial f_{t}\right|_{t=0}$ is horizontal for $f_{0}$. By [4, Theorem 2], if $f_{t}$ is a $C^{2}$ family of piecewise expanding $C^{2}$ unimodal maps with $f_{0}$

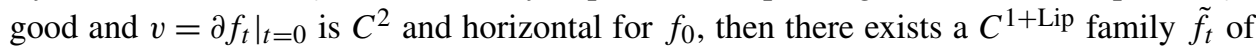
piecewise expanding $C^{2}$ unimodal maps in the topological class of $f_{0}$ so that $\tilde{f}_{t}=f_{0}$ and $\left.\partial_{t} f_{t}\right|_{t=0}=\left.\partial_{t} \tilde{f}_{t}\right|_{t=0}$.

We proved in [4, Lemma 2.2] that if $v: I \rightarrow \mathbb{R}$ is bounded then the twisted cohomological equation (TCE)

$$
v(x)=\alpha(f(x))-f^{\prime}(x) \alpha(x) \text { for all } x \in I, x \neq c,
$$

admits a unique bounded solution $\alpha$ satisfying $\alpha(c)=0$. This solution is obtained as follows: if $c$ is not in the forward orbit of $x$, set $M(x)=\infty$ and otherwise let $M(x)$ be the smallest integer $j \geq 0$ satisfying $f^{j}(x)=c$, then put

$$
\alpha(x)=-\sum_{i=0}^{M(x)-1} \frac{v\left(f^{i}(x)\right)}{\left(f^{i+1}\right)^{\prime}(x)} .
$$

The function $\alpha$ is called the infinitesimal conjugacy. Note that if $v$ is, in addition, $C^{2}$ and horizontal, then it follows from [4, Corollary 2.6, Theorem 2.8], see also [5, Theorem 2], that $\alpha$ is continuous. (Proposition 2.3 below states that $\alpha$ is continuous, in fact Hölder, if $v$ is Hölder and horizontal. If $v$ is $C^{0}$ and horizontal, then $\alpha$ should be continuous, approaching $v$ by Hölder continuous functions.)

If $u: I \rightarrow \mathbb{R}$ is Hölder, we denote its Hölder norm by $|u|_{\beta}$. Slightly abusing notation, we sometimes write $\partial_{t} f_{t}$ for $\left.\partial_{s} f_{s}\right|_{s=t}$, and similarly for other functions depending on $t$. 
2.2. Hölder smoothness of the infinitesimal conjugacy $\alpha$. A new result that we require throughout (see Lemmas 3.3 and 4.2) is the following.

PROPOSITION 2.3. (Smoothness of the infinitesimal conjugacy) Let $f \in \mathcal{U}^{2}$ be such that $c$ is not periodic. For any $\beta \in(0,1)$ there exist $C_{\beta}>0$ and $\mathcal{V}_{\beta}$ a neighbourhood of $f$ in $\mathcal{U}^{2}$ so that, for any $g \in \mathcal{V}_{\beta}$ and every $\beta$-Hölder $v: I \rightarrow \mathbb{R}$, with $v(-1)=v(1)=0$ and $J(g, v)=0$, the unique bounded function $\alpha$ (4) satisfying $\alpha(c)=0$ and $v(x)=\alpha$ $(g(x))-g^{\prime}(x) \alpha(x)$ for all $x \neq c$ is $\beta$-Hölder, with

$$
|\alpha|_{\beta} \leq C_{\beta}|v|_{\beta} .
$$

If the critical point of $f \in \mathcal{U}^{2}$ is periodic, the statement holds up to taking (for appropriate $\xi(\beta)>0$ )

$$
\mathcal{V}_{\beta}=\left\{g \mid\|g-f\|_{\mathcal{B}^{2}}<\xi(\beta), \exists \text { homeomorphism } h: I \rightarrow I \text { s.t. } g \circ h=h \circ f\right\} .
$$

In particular, if $f \in \mathcal{U}^{2}$ and $J(f, v)=0$ for some Lipschitz $v$ with $v(-1)=v(1)=0$, the function $\alpha$ solving (3) is $\beta$-Hölder for any $\beta<1$.

Remark 2.4. Buzzi [8] showed us a simple proof that if $h$ is a homeomorphism so that $h \circ f=g \circ h$, for two piecewise expanding $C^{1}$ unimodal maps $f$ and $g$, then $h$ is $\beta$-Hölder, for any $\beta<\log \left(\inf \left|g^{\prime}\right| / 2\right) / \log \left(2 \sup \left|f^{\prime}\right|\right)$. This fact neither implies nor is implied by Proposition 2.3.

Proof.

Step I. For any $\beta<1$, there exist a neighbourhood $\mathcal{V}_{\beta}$ of $f$ in $\mathcal{U}^{2}, \ell \geq 1$ and $\eta>0$ such that $\lambda=\left(\inf _{g \in \mathcal{V}_{\beta}} \inf _{x \neq c}\left|g^{\prime}(x)\right|\right)^{-1}<1$ and, for any $g \in \mathcal{V}_{\beta}$, letting $d_{1}<d_{2}<\cdots<d_{p}$ be the critical points of $g^{\ell}$, putting $d_{0}=-1, d_{p+1}=1$ and setting

$$
\theta=\max _{0 \leq i \leq p} \sup _{\substack{x, y \in\left(d_{i}, d_{i+1}\right) \\|x-y|<\eta}} \frac{\left|\left(g^{\ell}\right)^{\prime}(x)\right|^{\beta}}{\left|\left(g^{\ell}\right)^{\prime}(y)\right|},
$$

we have $2 \theta<1$.

Put $\Delta_{g}=\min _{0 \leq i \leq p}\left\{d_{i+1}-d_{i}\right\}$. Then $\inf _{g \in \mathcal{V}_{\beta}} \Delta_{g}>0$ if the critical point of $f$ is not periodic. Otherwise we have $\inf _{g \in \mathcal{V}_{\beta}} \Delta_{g}>0$, up to replacing $\mathcal{V}_{\beta}$ by a $\mathcal{B}^{2}$-neighbourhood of $f$ in its topological class. In particular, we can assume that $\eta<\inf _{g \in \mathcal{V}_{\beta}} \Delta_{g}$. From now on, we fix $\mathcal{V}_{\beta}, \ell \geq 1$, and $\eta>0$ as above.

Step II. We claim that it suffices to show the lemma for $g \in \mathcal{V}_{\beta}$ with a periodic critical point: indeed, if $g$ has a non-periodic critical point, then we consider $g_{t}=g+t w$ with $g_{t} \in \mathcal{U}^{2}, w \in \mathcal{B}^{2}, w(-1)=w(1)=0$, and $J(g, w) \neq 0$. By [5, Corollary 4.1], there exists a sequence $t_{n} \rightarrow 0$ such that each $g_{n}=g_{t_{n}}$ has a periodic critical point. In particular, $g_{n}$ converges to $g$ in the $\mathcal{U}^{2}$ topology. Then, by [5, Proposition 3.2] we have $\lim _{n \rightarrow \infty} J\left(g_{n}, v\right)=0$. Let $w_{n}$ be a $\beta$-Hölder function, with $w_{n}(-1)=w_{n}(1)=0$ and $\left|w_{n}\right|_{\beta} \leq 1$, such that $J\left(g_{n}, w_{n}\right)=1$. Set

$$
v_{n}=v-J\left(g_{n}, v\right) w_{n} .
$$

Then we have $J\left(g_{n}, v_{n}\right)=0$ and $\lim _{n \rightarrow \infty}\left|v_{n}-v\right|_{\beta}=0$. If the proposition holds for maps in $\mathcal{V}_{\beta}$ with a periodic turning point, the unique function $\alpha_{n}$ so that $\alpha_{n}(c)=0$ and 
$v_{n}(x)=\alpha_{n}\left(g_{n}(x)\right)-g_{n}^{\prime}(x) \alpha_{n}(x)$ for all $x \neq c$, satisfies $\left|\alpha_{n}\right|_{\beta} \leq C_{\beta}\left|v_{n}\right|_{\beta}$. We can choose a subsequence $\alpha_{n_{i}}$ converging in the sup norm to a function $\alpha$. It follows from the uniform convergence of $\alpha_{n_{i}}$ that $\alpha$ satisfies the TCE (3) for $g$ and $v$, and that $|\alpha|_{\beta} \leq C_{\beta}|v|_{\beta}$.

Step III. We assume from now on that $g \in \mathcal{V}_{\beta}$ has a periodic turning point. The proof will be via an 'infinitesimal pull-back' argument.

First, since $J(g, v)=0$, it is easy to see that there exists a $\beta$-Hölder function $\alpha_{0}: I \rightarrow \mathbb{R}$ with $\alpha_{0}(-1)=\alpha_{0}(1)=\alpha_{0}(c)=0, \alpha_{0}(g(c))=v(c)$, and

$$
v(x)=\alpha_{0}(g(x))-g^{\prime}(x) \alpha_{0}(x) \text { for every } x \neq c \text { in the (finite) forward orbit of } c .
$$

Second, we define by induction continuous functions $\alpha_{i}: I \rightarrow \mathbb{R}$, for $i \geq 1$, such that $\alpha_{i}(-1)=\alpha_{i}(1)=\alpha_{i}(c)=0, \alpha_{i}(g(c))=v(g(c))$, that

$$
v(x)=\alpha_{i}(g(x))-g^{\prime}(x) \alpha_{i}(x) \text { for every } x \neq c \text { in the (finite) forward orbit of } c,
$$

and, in addition,

$$
v(x)=\alpha_{i-1}(g(x))-g^{\prime}(x) \alpha_{i}(x) \text { for all } x \neq c .
$$

Indeed, suppose that we have defined $\alpha_{i}$, for $0 \leq i \leq n$. Set $\alpha_{n+1}(c)=0$, and

$$
\alpha_{n+1}(x)=\frac{\alpha_{n}(g(x))-v(x)}{g^{\prime}(x)}, \quad x \neq c .
$$

Clearly, $\alpha_{n+1}(-1)=\alpha_{n+1}(1)=0$, and (7) holds for $i=n+1$. Thus, since $v(x)=$ $\alpha_{n}(g(x))-g^{\prime}(x) \alpha_{n}(x)$ for every $x \neq c$ in the forward orbit of $c$, we find $\alpha_{n}(x)=\alpha_{n+1}(x)$ for each $x \neq c$ in the forward orbit of $c$. Since $\alpha_{n}(c)=\alpha_{n+1}(c)=0$, we conclude that (6) holds for $i=n+1$, and $\alpha_{n+1}(g(x))=v(x)$. Last, but not least, $\alpha_{n+1}$ is continuous on $I$ because $\alpha_{n}(g(x))=v(x)$.

Third, if $x$ is not a critical point of $g^{j}$, we set $v_{0}(x)=0$, and

$$
v_{j}(x)=\sum_{i=0}^{j-1}\left(g^{j-1-i}\right)^{\prime}\left(g^{i+1}(x)\right) v\left(g^{i}(x)\right), \quad j \geq 1 .
$$

Recalling the notation $\ell,\left\{d_{i}\right\}$, from Step I, it is easy to see that

$$
v_{\ell}(x)=\alpha_{j \ell}\left(g^{\ell}(x)\right)-\left(g^{\ell}\right)^{\prime}(x) \alpha_{(j+1) \ell}(x) \quad \text { for all } x \notin\left\{d_{1}, \ldots d_{p}\right\}, \text { for all } j \geq 0 .
$$

For $\ell \geq 2$ the function $v_{\ell}$ may have jump discontinuities at the critical points $d_{i}$ of $g^{\ell}$, but it is $\beta$-Hölder in the connected components of $I \backslash\left\{d_{1}, \ldots, d_{p}\right\}$.

Finally, we use the iterated TCE (8) to show that there exists $C_{\beta}<\infty$ so that, for all $g \in \mathcal{V}_{\beta}$ with a periodic turning point and all $\beta$-Hölder $v$ with $J(g, v)=0$ (and $v(-1)$ $=v(1)=0)$, there exists $j_{0} \geq 0$ so that $\left|\alpha_{j \ell}\right|_{\beta} \leq C_{\beta}|v|_{\beta}$ for all $j \geq j_{0}$. In view of this, for $j \geq 0$, set $K_{j}^{0}=\sup _{I}\left|\alpha_{j \ell}\right|, L^{0}=\sup _{I}\left|v_{\ell}\right|$,

$$
K_{j}^{\beta}=\sup _{x \neq y} \frac{\left|\alpha_{j \ell}(x)-\alpha_{j \ell}(y)\right|}{|x-y|^{\beta}}, \quad \widehat{K}_{j}^{\beta}=\max _{\substack { 0 \leq i \leq p \\
\begin{subarray}{c}{x \neq y,|x-y|<\eta \\
x, y \in\left(d_{i}, d_{i+1}\right){ 0 \leq i \leq p \\
\begin{subarray} { c } { x \neq y , | x - y | < \eta \\
x , y \in ( d _ { i } , d _ { i + 1 } ) } }\end{subarray}} \frac{\left|\alpha_{j \ell}(x)-\alpha_{j \ell}(y)\right|}{|x-y|^{\beta}},
$$

and

$$
L^{\beta}=\max _{0 \leq i \leq p} \sup _{\substack{x \neq y \\ x, y \in\left(d_{i}, d_{i+1}\right)}} \frac{\left|v_{\ell}(x)-v_{\ell}(y)\right|}{|x-y|^{\beta}}, \quad D=\max _{0 \leq i \leq p} \sup _{x, y \in\left(d_{i}, d_{i+1}\right)} \frac{\left|\left(g^{\ell}\right)^{\prime \prime}(x)\right|}{\left|\left(g^{\ell}\right)^{\prime}(y)\right|^{2}} .
$$


Clearly, $\max \left(L^{0}, L^{\beta}\right) \leq \widetilde{C}_{\beta}|v|_{\beta}$ for all $g$ and $v$ under consideration, and we have

$$
\frac{\left|\alpha_{(j+1) \ell}(x)-\alpha_{(j+1) \ell}(y)\right|}{|x-y|^{\beta}} \leq 2 \eta^{-\beta} K_{j+1}^{0} \quad \text { if }|x-y| \geq \eta \text {, for all } j \geq 0 .
$$

Therefore, recalling the definition of $\lambda$ and (5) from Step I, it suffices to show that

$$
K_{j+1}^{0} \leq \lambda^{\ell}\left(K_{j}^{0}+L^{0}\right) \quad \text { and } \quad \widehat{K}_{j+1}^{\beta} \leq\left(L^{0}+K_{j}^{0}\right) D+\lambda^{\ell} L^{\beta}+\theta K_{j}^{\beta} \quad \text { for all } j \geq 0 .
$$

Indeed, continuity of $\alpha_{(j+1) \ell}$ together with (9) (recall also that $\eta<\inf _{g} \Delta_{g}$, so that if $|x-y|<\eta$ then $[x, y]$ contains at most one point $d_{i}$ ) imply

$$
K_{j+1}^{\beta} \leq \max \left(2 \eta^{-\beta} K_{j+1}^{0}, 2 \widehat{K}_{j+1}^{\beta}\right) .
$$

The above bound together with (10) yield $E_{\beta}<\infty$ so that, for all $g \in \mathcal{V}_{\beta}$ with a periodic turning point, and all $\beta$-Hölder $v$ with $J(g, v)=0, v(-1)=v(1)=0$, there exists $j_{0} \geq 0$ so that

$$
K_{j+1}^{\beta} \leq E_{\beta}|v|_{\beta}+2 \theta K_{j}^{\beta} \quad \text { for all } j \geq j_{0} .
$$

Since $2 \theta<1$, we conclude by a geometric series, taking larger $j_{0}$ if necessary.

It remains to show (10). We concentrate on the second bound (the first is easier and left to the reader). Let $x, y \in\left(d_{i}, d_{i+1}\right)$ satisfy $|x-y|<\eta$. Then (8) implies (since $g \in \mathcal{U}^{2}$, the function $\left(g^{\ell}\right)^{\prime}$ is $C^{1}$ in the intervals of monotonicity of $g^{\ell}$ )

$$
\begin{aligned}
\left|\alpha_{(j+1) \ell}(x)-\alpha_{(j+1) \ell}(y)\right| \leq & \left|v_{\ell}(x)+\alpha_{j \ell}\left(g^{\ell}(x)\right)\right|\left|\frac{1}{\left(g^{\ell}\right)^{\prime}(x)}-\frac{1}{\left(g^{\ell}\right)^{\prime}(y)}\right| \\
& +\frac{\left|v_{\ell}(x)-v_{\ell}(y)\right|+\left|\alpha_{j \ell}\left(g^{\ell}(x)\right)-\alpha_{j \ell}\left(g^{\ell}(y)\right)\right|}{\left|\left(g^{\ell}\right)^{\prime}(y)\right|} \\
\leq & \left(L^{0}+K_{j}^{0}\right) \frac{\sup _{[x, y]}\left|\left(g^{\ell}\right)^{\prime \prime}\right|}{\inf _{[x, y]}\left|\left(g^{\ell}\right)^{\prime}\right|^{2}}|x-y| \\
& +\frac{L^{\beta}+\sup _{[x, y]}\left|\left(g^{\ell}\right)^{\prime}\right|^{\beta} K_{j}^{\beta}}{\inf _{[x, y]}\left|\left(g^{\ell}\right)^{\prime}\right|}|x-y|^{\beta} \\
\leq & \left(L^{0}+K_{j}^{0}\right) D|x-y|+\left(\lambda^{\ell} L^{\beta}+\theta K_{j}^{\beta}\right)|x-y|^{\beta} .
\end{aligned}
$$

Step IV. Defining $\tilde{\alpha}_{n}=(1 / n) \sum_{j=0}^{n-1} \alpha_{j \ell}$, we can choose a subsequence $\tilde{\alpha}_{n_{i}}$ converging uniformly on $I$ to a function $\tilde{\alpha}$ satisfying $|\tilde{\alpha}|_{\beta} \leq C_{\beta}|v|_{\beta}$. By (8),

$$
v_{\ell}(x)=\tilde{\alpha}\left(g^{\ell}(x)\right)-\left(g^{\ell}\right)^{\prime}(x) \tilde{\alpha}(x) \text { for all } x \notin\left\{d_{1}, \ldots, d_{p}\right\} .
$$

Let $\alpha: I \rightarrow \mathbb{R}$ be the unique bounded solution vanishing at $c$ to the TCE (3) for $g$ and $v$, as in (4). Then

$$
v_{\ell}(x)=\alpha\left(g^{\ell}(x)\right)-\left(g^{\ell}\right)^{\prime}(x) \alpha(x) \text { for all } x \notin\left\{d_{1}, \ldots, d_{p}\right\} .
$$

Since $\tilde{\alpha}$ is continuous (11) and (12), imply $\alpha=\tilde{\alpha}$. We have proved that $|\alpha|_{\beta} \leq C_{\beta}|v|_{\beta}$, for all $g \in \mathcal{V}_{\beta}$ with a periodic turning point, and thus the proposition. 
2.3. Banach spaces of functions of bounded variation. We consider the Banach space of functions of bounded variation

$$
B V=\{\varphi: \mathbb{R} \rightarrow \mathbb{C} \mid \operatorname{var}(\varphi)<\infty, \operatorname{supp}(\varphi) \subset I\} / \sim,
$$

endowed with the norm $\|\varphi\|_{B V}=\inf _{\psi \sim \varphi} \operatorname{var}(\psi)$, where var denotes total variation, and $\varphi_{1} \sim \varphi_{2}$ if the bounded functions $\varphi_{1}, \varphi_{2}$ differ on an at most countable set. In addition, for $1 \leq p<\infty$ we work with the Banach space of functions of bounded $p$-variation (used in interval dynamics by Wong [22])

$$
B V_{p}=\left\{\varphi: \mathbb{R} \rightarrow \mathbb{C} \mid \operatorname{var}_{p}(\varphi)<\infty, \operatorname{supp}(\varphi) \subset I\right\} / \sim,
$$

where

$$
\operatorname{var}_{p}(\varphi)=\sup _{x_{1}<x_{2}<\cdots<x_{n}}\left(\sum_{i=1}^{n}\left|\varphi\left(x_{i+1}\right)-\varphi\left(x_{i}\right)\right|^{p}\right)^{1 / p},
$$

the supremum ranging over all ordered finite subsets of $\mathbb{R}$. Note that $\operatorname{var}_{1}=$ var and $B V=B V_{1}$. Wong [22] does not quotient by the equivalence relation $\varphi_{1} \sim \varphi_{2}$, but his results remain unchanged if we consider elements in $B V_{p}$ modulo $\sim$ (a function in $B V_{p}$ is continuous except on an at most countable set, see also [15, Lemmas 1.4.a and 2.7] and [7]). Note that for each $p \geq 1$ there is $C \geq 1$ so that $|\varphi|_{\infty} \leq C\|\varphi\|_{B V_{p}}$ for all $\varphi$, and if $\varphi$ is $1 / p$-Hölder, then $\|\varphi\|_{B V_{p}} \leq|\varphi|_{1 / p}$. In addition, we claim that for any $K>0$ and $p>1$, and for every $\varphi$ with $\|\varphi\|_{C^{1}} \leq K$

$$
\|\varphi\|_{C^{1 / p}} \leq K^{1 /(p-1)}\|\varphi\|_{B V_{p}} .
$$

To prove (13), it suffices to show that if $\|\varphi\|_{C^{1 / p}}=1$, then $\|\varphi\|_{B V_{p}} \geq K^{-1 /(p-1)}$. By the mean value theorem, there exist $x_{i} \leq y_{i} \leq x_{i+1}$ so that

$$
1=\|\varphi\|_{C^{1 / p}}=\left|\varphi\left(x_{i+1}\right)-\varphi\left(x_{i}\right)\right|\left|x_{i+1}-x_{i}\right|^{-1 / p}=\left|\varphi^{\prime}\left(y_{i}\right)\right|\left|x_{i+1}-x_{i}\right|^{1-1 / p},
$$

and, in particular, $\left|x_{i+1}-x_{i}\right| \geq K^{-p /(p-1)}$. By definition, and the mean value theorem again,

$$
\|\varphi\|_{B V_{p}} \geq\left|\varphi\left(x_{i+1}\right)-\varphi\left(x_{i}\right)\right|=\left|\varphi^{\prime}\left(y_{i}\right)\right|\left|x_{i+1}-x_{i}\right| \geq K^{-1 /(p-1)}\left|\varphi^{\prime}\left(y_{i}\right)\right|\left|x_{i+1}-x_{i}\right|^{1-1 / p} .
$$

This ends the proof of (13). Finally,

$$
\left\|\varphi_{1} \varphi_{2}\right\|_{B V_{p}} \leq 2\left\|\varphi_{1}\right\|_{B V_{p}}\left\|\varphi_{2}\right\|_{B V_{p}} \quad \text { for all } p \geq 1
$$

and

$$
\|\varphi \circ h\|_{B V_{p}}=\|\varphi\|_{B V_{p}} \quad \text { for any homeomorphism } h: I \rightarrow I \text { and all } p \geq 1 .
$$

Remark 2.5. The reason we consider spaces $B V_{p}$ for $p \neq 1$ is because we are concerned with differentiability in the $t$-parameter and we have to deal with derivatives $\partial_{t}(\psi \circ$ $\left.h_{t}\right)\left.\right|_{t=0}=\left.\psi^{\prime} \cdot \partial_{t} h_{t}\right|_{t=0}$ or $\left.\partial_{t}\left(f_{t}^{\prime} \circ h_{t}\right)\right|_{t=0}=\left.f_{0}^{\prime \prime} \partial_{t} h_{t}\right|_{t=0}+v^{\prime}$, where $v^{\prime}=\left.\partial_{t} f_{t}^{\prime}\right|_{t=0}$ is $C^{1}$, but $\left.\partial_{t} h_{t}\right|_{t=0}$ does not belong to $B V$ in general. We shall see, however, that Proposition 2.3 implies that $\alpha=\left.\partial_{t} h_{t}\right|_{t=0}$ lies in $B V_{p}$ for all $p>1$. 


\section{Weak differentiability of the SRB via the pressure}

The main result of this section (Theorem 3.1) says that for any $j \geq 1$, if $f_{t}$ is a $C^{j+1}$ family of piecewise expanding $C^{j+2}$ unimodal maps in the topological class of $f_{0}$, then $R(t)=\int \psi d \mu_{t}$ is $C^{j}$ if $\psi$ is $C^{j+\mathrm{Lip}}$. Even if $j=1$, this is a new result ([4, Theorem 5.1] only gives differentiability at $t=0$ ). The argument is based on the topological pressure of the potential $(s, t) \mapsto-\log \left|f_{t}^{\prime} \circ h_{t}\right|+s\left(\psi \circ h_{t}\right)$ for the map $f_{0}$. It is simple, but does not give the formula for $\left.\partial_{t} R(t)\right|_{t=0}$ (or higher order derivatives). Using the linear response formula from [4, Theorem 5.1] or Theorem 4.1 below, Theorem 3.1 will imply Corollary 4.4 .

THEOREM 3.1. For any integer $j \geq 1$, if $f_{t}$ is a $C^{j+1}$ family of piecewise expanding $C^{j+2}$ unimodal maps in the topological class of a mixing map $f_{0}$, then there is $\hat{\epsilon}>0$ so that for any $C^{j+\text { Lip }}$ function $\psi$ the map $R(t)=\int \psi \rho_{t} d x$ is $C^{j}$ in $(-\hat{\epsilon}, \hat{\epsilon})$.

As an immediate corollary of Theorem 3.1 and Proposition A.1, we recover the first claim of [4, Theorem 5.1] if $\psi$ is $C^{1+\text { Lip }}$ (we do not need the assumption $\left.\partial f_{t}\right|_{t=0}=X \circ f_{0}$ used in [4, Theorem 5.1]).

COROLlary 3.2. Assume that $f_{t}$ is a $C^{2}$ family of piecewise expanding $C^{3}$ unimodal maps, where $f_{0}$ is a good mixing map. If $f_{t}$ is tangent to the topological class of $f_{0}$, then for any $C^{1+\text { Lip }}$ function $\psi: I \rightarrow \mathbb{C}$, the map $R(t)=\int \psi d \mu_{t}$ is differentiable at $t=0$.

Proof of Theorem 3.1. Fix $\psi \in C^{1+\mathrm{Lip}}$, recall the notation $h_{t}$ from (1), put

$$
g_{s, t}(y)=\frac{\exp \left(s \psi\left(h_{t}(y)\right)\right)}{\left|f_{t}^{\prime}\left(h_{t}(y)\right)\right|}, \quad y \in I \backslash\{c\},
$$

and consider the transfer operators

$$
\widetilde{\mathcal{L}}_{s, t} \varphi(x)=\sum_{f(y)=x} g_{s, t}(y) \varphi(y), \quad \mathcal{L}_{s, t} \varphi(x)=\sum_{f_{t}(y)=x} e^{s \psi(y)} \frac{\varphi(y)}{\left|f_{t}^{\prime}(y)\right|} .
$$

Then since $\left|f_{t}^{\prime}\right|$ is the Jacobian of $f_{t}$ with respect to Lebesgue measure $d x$ the operator $\mathcal{L}_{t}=\mathcal{L}_{0, t}$ is just the usual transfer operator for $f_{t}$. In particular, the change of variable formula implies $\mathcal{L}_{t}^{*}(d x)=d x$ for all small $t$. Also, the main theorem of [22] applied to $\mathcal{L}_{t}$ gives $p_{0}>1$ (depending on $f_{0}$ through inf $\left|f_{0}^{\prime}\right|$ and sup $\left|f_{0}^{\prime}\right|$ ) so that for any $p \in\left[1, p_{0}\right.$ ) there exists $\epsilon_{p}>0$ so that for all $|t|<\epsilon_{p}$ the operator $\mathcal{L}_{t}$ acting on $B V_{p}$ has spectral radius 1 , essential spectral radius $<1$, and 1 is the only eigenvalue of modulus 1 and is simple (i.e. $\mathcal{L}_{t}$ has a spectral gap). Furthermore, the fixed vector $\rho_{t}$ is strictly positive on $\left[c_{2}, c_{1}\right]$. The fixed vector $v_{t}$ of $\mathcal{L}_{t}^{*}$ is $d x$, and we normalize so that $\int \rho_{t} d v_{t}=1$ and $v_{t}(I)=1$. (Of course, $\mu_{t}=\rho_{t} d x$ is just the SRB measure of $f_{t}$.)

The transfer operator $\widetilde{\mathcal{L}}_{s, t}$ is conjugated to $\mathcal{L}_{s, t}$ via

$$
\widetilde{\mathcal{L}}_{s, t}\left(\varphi \circ h_{t}\right)=\mathcal{L}_{s, t}(\varphi) \circ h_{t} .
$$

Therefore, (15) (which says that $h_{t}$ is an isometry of $B V_{p}$ ) implies that the spectra of $\widetilde{\mathcal{L}}_{s, t}$ and $\mathcal{L}_{s, t}$ on $B V_{p}$ coincide. In particular, the operator $\widetilde{\mathcal{L}}_{t}=\widetilde{\mathcal{L}}_{0, t}$ on $B V_{p}$ has a simple eigenvalue at 1 , for the fixed point $\tilde{\rho}_{t}=\rho_{t} \circ h_{t}$, and the rest of its spectrum lies in a disc of strictly smaller radius. The fixed point of $\widetilde{\mathcal{L}}_{t}^{*}$ is the measure $v_{t}$ defined by

$$
\int \varphi d x=\int \varphi \circ h_{t} d \nu_{t}
$$


(By definition, $v_{t}$ is a probability measure and $\int \tilde{\rho}_{t} d v_{t}=1$.)

We view $\mathcal{L}_{s, t}$ as a perturbation of $\mathcal{L}_{t}$, writing

$$
\mathcal{L}_{s, t}(\varphi)=\mathcal{L}_{t}\left(e^{s \psi(y)} \varphi\right)
$$

Since $\psi$ and the exponential are $C^{1+\mathrm{Lip}}$, the norm $\left\|e^{s \psi(y)}-1\right\|_{B V_{1}}$ tends to zero as $s \rightarrow 0$ (uniformly in $t$ ). Therefore, applying classical perturbation theory [11], the operators $\mathcal{L}_{s, t}$ (or, equivalently, $\widetilde{\mathcal{L}}_{s, t}$ ) on $B V_{p}$ have a real positive simple maximal $\dagger$ eigenvalue $\lambda_{s, t}>0$ with a spectral gap, uniformly in $(s, t)$ close enough to $(0,0)$.

Consider first the case $j=1$. Setting $\mathcal{P}_{s, t}(\varphi)=\left(g_{s, t} / g_{0,0}\right) \varphi$, Lemma 3.3 below implies that the map $s \mapsto \mathcal{P}_{s, t}$ is $C^{1}$ from $\mathbb{R}$ to the Banach space of $C^{1}$ maps from $\{|t|<\epsilon\}$ to bounded operators on $B V_{p}$, and

$$
\left.\partial_{s} \mathcal{P}_{s, t}\right|_{s=u}=\left(\psi \circ h_{t}\right) \mathcal{P}_{u, t} \quad \text { for all } u \in \mathbb{R} .
$$

Therefore, $s \mapsto \widetilde{\mathcal{L}}_{s, t}=\mathcal{L}_{0} \circ \mathcal{P}_{s, t}$ is $C^{1}$ from $\mathbb{R}$ to the Banach space of $C^{1}$ maps from $\{|t|<\epsilon\}$ to bounded operators on $B V_{p}$, and

$$
\left.\partial_{s} \widetilde{\mathcal{L}}_{s, t}\right|_{s=u}(\varphi)=\widetilde{\mathcal{L}}_{u, t}\left(\left(\psi \circ h_{t}\right) \varphi\right) \quad \text { for all } u \in \mathbb{R} .
$$

We are thus in a position to apply classical perturbation theory of an isolated simple eigenvalue (see [11, Ch. VII.1.3] for the analytic case, see e.g. [3, Lemma 3.2] for the differentiable setting). It follows, on the one hand, that in a neighbourhood of $(0,0)$ the maximal eigenvalue $\lambda_{s, t}>0$ of $\widetilde{\mathcal{L}}_{s, t}$ acting on $B V_{p}$ is a $C^{1}$ function of $s$ to the space of $C^{1}$ maps from $\{|t|<\epsilon\}$ to $\mathbb{R}$. On the other hand, by 'tedious but straightforward calculations' and [11, Ch. VII.1.5 and II.2.2], (to quote [17, (5.2)]), we have

$$
\left.\partial_{s}\left(\log \lambda_{s, t}\right)\right|_{s=0}=\int \psi \circ h_{t} \tilde{\rho}_{t} d v_{t}=\int \psi d \mu_{t}
$$

(use that $\tilde{\rho}_{t}$ and $v_{t}$ are the fixed eigenvectors of $\widetilde{\mathcal{L}}_{0, t}$ and its dual). Since $t \mapsto \partial_{S}$ $\left.\left(\log \lambda_{s, t}\right)\right|_{s=0}$ is a $C^{1}$ function in a neighbourhood of zero, we have proved Theorem 3.1 in the case $j=1$. If $j \geq 2$, apply Lemma 3.4 instead of Lemma 3.3.

The following result is the key ingredient in the proof of Theorem 3.1, its proof hinges on Proposition 2.3 and [4, Proposition 2.4].

LEMMA 3.3. Let $f_{t}$ be a $C^{2}$ family of piecewise expanding $C^{3}$ unimodal maps in the topological class of $f_{0}$. For any $p>1$ there exists $\epsilon_{p}>0$ so that for any $\psi: I \rightarrow \mathbb{R}$ which is $C^{1+\mathrm{Lip}}$, the map $s \mapsto g_{s, t}$ defined by (16) is $C^{1}$ from $\mathbb{R}$ to the Banach space of $C^{1}$ maps from $\left\{|t|<\epsilon_{p}\right\}$ to $B V_{p}$. In addition, recalling the notation (1),

$$
\left.\partial_{s} g_{s, t}\right|_{s=u}=\left(\psi \circ h_{t}\right) g_{u, t} \quad \text { for all } u \in \mathbb{R} .
$$

In fact, $s$-analyticity holds in Lemma 3.3, but we do not need this.

Proof of Lemma 3.3. Fix $p>1$. For every $x \neq c$, all small $t$, and all $s_{1}, s_{2}$ in $\mathbb{R}$, we have

$$
g_{s_{1}, t}(x)-g_{s_{2}, t}(x)=g_{s_{2}, t}(x) \sum_{k=1}^{\infty} \frac{\left(s_{1}-s_{2}\right)^{k}}{k !}\left(\psi\left(h_{t}(x)\right)\right)^{k} .
$$

$\dagger$ Of course, $\log \lambda_{s, t}$ is the topological pressure of $\log g_{s, t}$. 
So, to prove both differentiability and (22), it suffices to see that the maps

$$
t \mapsto \frac{1}{k !}\left(\psi \circ h_{t}\right)^{k} g_{s, t}, \quad k \geq 0,
$$

are $C^{1}$ from a neighbourhood of 0 to $B V_{p}$, uniformly in $k$ and in $s$ in any compact set $K \subset \mathbb{R}$.

In view of this, we first study the maps $t \mapsto h_{t}(x)$. By [4, Proposition 2.4], there exists $\tilde{\epsilon}>0$ so that the set of maps $\left\{t \mapsto h_{t}(x), x \in I\right\}$ is bounded in $C^{1+\operatorname{Lip}}([-\tilde{\epsilon}, \tilde{\epsilon}])$. Differentiating with respect to $t$ the equation $h_{t} \circ f_{0}=f_{t} \circ h_{t}$, and setting $\alpha_{t}=\partial_{t} h_{t} \circ h_{t}^{-1}$, we obtain

$$
\alpha_{t}\left(f_{t}(c)\right)=\partial_{t} f_{t}(c), \quad \partial_{t} f_{t}(x)=\alpha_{t}\left(f_{t}(x)\right)-f_{t}^{\prime}(x) \alpha_{t}(x) \text { for all } x \neq c,|x|<\tilde{\epsilon} .
$$

Since $\alpha_{t}(c)=0$ this implies $J\left(f_{t}, \partial_{t} f_{t}\right)=0$ for $|t|<\tilde{\epsilon}$ (recall (4)), so, for any fixed

$$
\beta \in(1 / p, 1) \quad \text { (we may and do assume also that } \beta<1 / \sqrt{p} \text { ), }
$$

Proposition 2.3 gives $C$ and $\epsilon_{p}>0$ so that

$$
\left|\alpha_{t}\right|_{\beta} \leq C \text { for all }|t|<\epsilon_{p} .
$$

Let $\alpha_{t}^{\eta}$ be the $\eta$-regularization (in the variable $x$ ) of $\alpha_{t}$, that is, the convolution $\alpha_{t}^{\eta}(x)$ $=\int \alpha_{t}(y) \kappa_{\eta}(x-y) d y$ of $\alpha_{t}$ with a convolution kernel $\kappa_{\eta}(x)=\eta^{-1} \kappa(x / \eta)$, where the $C^{\infty}$ function $\kappa: \mathbb{R} \rightarrow \mathbb{R}_{+}$is supported in $[-1,1]$, and $\int \kappa(x) d x=1$. Note for further use that (24) gives $\widetilde{C}$ so that, for all $|t|<\epsilon_{p}$,

$$
\left\|\alpha_{t}^{\eta}\right\|_{C^{1+1 / p}} \leq \frac{\widetilde{C}}{\eta^{1+1 / p-\beta}}, \quad\left\|\alpha_{t}^{\eta}\right\|_{\beta} \leq \widetilde{C}, \quad\left|\alpha_{t}^{\eta}-\alpha_{t}\right|_{1 / p} \leq \widetilde{C} \eta^{\beta-1 / p} \quad \text { for all } \eta \in(0,1) .
$$

We now consider $t \mapsto g_{s, t}$. For $x \neq c$, we have

$$
\partial_{t} g_{s, t}(x)=e^{s \psi\left(h_{t}(x)\right)}\left[\frac{\psi^{\prime}\left(h_{t}(x)\right) \alpha_{t}\left(h_{t}(x)\right)}{\left|f_{t}^{\prime}\left(h_{t}(x)\right)\right|}-\frac{\partial_{t}\left(\left|f_{t}^{\prime}\left(h_{t}(x)\right)\right|\right)}{\left|f_{t}^{\prime}\left(h_{t}(x)\right)\right|^{2}}\right],
$$

where

$$
\partial_{t}\left(\left|f_{t}^{\prime}\left(h_{t}(x)\right)\right|\right)=-\operatorname{sgn}(x)\left(f_{t}^{\prime \prime}\left(h_{t}(x)\right) \alpha_{t}\left(h_{t}(x)\right)+\partial_{t} f_{t}^{\prime}\left(h_{t}(x)\right)\right) .
$$

We claim that the function $x \mapsto \partial_{t} g_{s, t}(x)$ has bounded $B V_{1 / \beta}$ norm, uniformly in $s \in K$ and $|t|<\epsilon_{p}$. Indeed, decomposing

$$
\partial_{t} g_{s, t}=b_{s, t} \circ h_{t},
$$

note that each $h_{t}: I \rightarrow I$ is a homeomorphism leaving both $[-1, c]$ and $[c, 1]$ invariant, while $b_{s, t}$ is $\beta$-Hölder on $[-1, c)$ and $(c, 1]$, uniformly in $s \in K$ and $|t|<\epsilon_{p}$ (because $\psi^{\prime}$ is $C^{\beta}, f_{t}$ is a $C^{2}$ family of $C^{3}$ maps $\dagger$, and $\alpha_{t}$ is $\beta$-Hölder, uniformly in $|t|<\epsilon_{p}$ ), and $\sup _{s \in K,|t|<\epsilon_{p}}\left|b_{s, t}\left(c_{+}\right)-b_{s, t}\left(c_{-}\right)\right|<\infty\left(\operatorname{using} \sup _{|t|<\epsilon_{p}}\left\|f_{t}\right\|_{\mathcal{B}^{2+\beta}}<\infty\right)$.

To conclude, it suffices to prove that our candidate $b_{s, t} \circ h_{t} \in B V_{p}$ is really the $t$-derivative of $g_{s, t}$ (uniformly in $s$ ), that is,

$$
\lim _{t_{2} \rightarrow t_{1}} \sup _{s \in K}\left\|\frac{g_{s, t_{2}}-g_{s, t_{1}}}{t_{2}-t_{1}}-b_{s, t_{1}} \circ h_{t_{1}}\right\|_{B V_{p}}=0 \text { for all }\left|t_{1}\right|<\epsilon_{p},
$$

$\dagger$ This implies, in particular, that $x \mapsto \partial_{t} f_{t}$ is $C^{2}$ in $x$, uniformly in $t$ and $\partial_{x} \partial_{t} f_{t}=\partial_{t} f_{t}^{\prime}$. 
and that this derivative is continuous in $t$ (uniformly in $s$ ), that is,

$$
\lim _{\tilde{t} \rightarrow t_{1}} \sup _{t_{2} \in\left[t_{1}, \tilde{t}\right]} \sup _{s \in K}\left\|b_{s, t_{2}} \circ h_{t_{2}}-b_{s, t_{1}} \circ h_{t_{1}}\right\|_{B V_{p}}=0 \quad \text { for all }\left|t_{1}\right|<\epsilon_{p}
$$

We first prove (29). Decomposing

$$
b_{s, t_{2}} \circ h_{t_{2}}-b_{s, t_{1}} \circ h_{t_{1}}=\left(b_{s, t_{2}}-b_{s, t_{1}}\right) \circ h_{t_{2}}+b_{s, t_{1}} \circ h_{t_{2}}-b_{s, t_{1}} \circ h_{t_{1}},
$$

we focus first on the second term in the right-hand side of (30). Let $\delta>0$ be such that $f_{t}^{\prime}, f_{t}^{\prime \prime}$ and $\partial_{t} f_{t}^{\prime}$ restricted to $[-1, c]$ and $[c, 1]$, respectively, extend to $C^{1}$ functions of $x$ on $[-1-\delta, c+\delta]$ and $[c-\delta, 1+\delta]$, respectively, for all $|t|<\epsilon_{p}$. Denote by $b_{s, t}^{\eta,-}$ the function obtained from $b_{s, t}$ by substituting $\alpha_{t}$ with $\alpha_{t}^{\eta}$, and also $\psi^{\prime}$, and the extensions to $[-1-\delta, c+\delta]$ of $\left.f_{t}^{\prime \prime}\right|_{[-1, c]},\left.\partial_{t} f_{t}^{\prime}\right|_{[-1, c]}$, with their $x$-convolutions with $\kappa_{\eta}$, for small $\eta>0$ (to be determined later). Define $b_{s, t}^{\eta,+}$ similarly, using $[c-\delta, 1+\delta]$, and set $b_{s, t}^{\eta}(x)=b_{s, t}^{\eta,+}(x)$ if $x>c$ and $=b_{s, t}^{\eta,-}(x)$ if $x<c$. Since $\beta<1$ and $\psi^{\prime}$ is Lipschitz, it is easy to see that there exists $\widehat{C}>0$ so that for all $\eta \in(0,1)$

$$
\max \left(\sup _{s \in K}\left|\left(\left.b_{s, t_{1}}^{\eta}\right|_{(-\infty, c)}\right)^{\prime}\right|_{1 / p}, \sup _{s \in K}\left|\left(\left.b_{s, t_{1}}^{\eta}\right|_{(c, \infty)}\right)^{\prime}\right|_{1 / p}\right) \leq \frac{\widehat{C}}{\eta^{1+1 / p-\beta}} \quad \text { for all }\left|t_{1}\right|<\epsilon_{p} .
$$

(Use the first two estimates of (25), and the analogous bounds for the regularizations of $\psi^{\prime}$ and $f_{t}^{\prime \prime}, \partial_{t} f_{t}^{\prime}$.) Therefore, by the fundamental theorem of calculus and the Hölder (or Jensen) inequality, there exists $\bar{C}>0$ so that for all $s \in K$, all $\left|t_{1}\right|<\epsilon_{p},\left|t_{2}\right|<\epsilon_{p}$, all $\eta \in(0,1)$, and any $x_{0}<x_{1}<\cdots<x_{N} \leq c$,

$$
\begin{aligned}
& \sum_{i=0}^{N-1}\left|b_{s, t_{1}}^{\eta}\left(h_{t_{2}}\left(x_{i}\right)\right)-b_{s, t_{1}}^{\eta}\left(h_{t_{1}}\left(x_{i}\right)\right)-b_{s, t_{1}}^{\eta}\left(h_{t_{2}}\left(x_{i+1}\right)\right)+b_{s, t_{1}}^{\eta}\left(h_{t_{1}}\left(x_{i+1}\right)\right)\right|^{p} \\
& \quad=\sum_{i}\left|\int_{t_{1}}^{t_{2}} \partial_{t}\left(b_{s, t_{1}}^{\eta}\left(h_{t}\left(x_{i}\right)\right)\right) d t-\int_{t_{1}}^{t_{2}} \partial_{t}\left(b_{s, t_{1}}^{\eta}\left(h_{t}\left(x_{i+1}\right)\right)\right) d t\right|^{p} \\
& \quad \leq \sum_{i} \int_{t_{1}}^{t_{2}}\left|\left(b_{s, t_{1}}^{\eta}\right)^{\prime}\left(h_{t}\left(x_{i}\right)\right) \alpha_{t}\left(h_{t}\left(x_{i}\right)\right)-\left(b_{s, t_{1}}^{\eta}\right)^{\prime}\left(h_{t}\left(x_{i+1}\right)\right) \alpha_{t}\left(h_{t}\left(x_{i+1}\right)\right)\right|^{p} d t \\
& \quad=\int_{t_{1}}^{t_{2}} \sum_{i}\left|\left(b_{s, t_{1}}^{\eta}\right)^{\prime}\left(h_{t}\left(x_{i}\right)\right) \alpha\left(h_{t}\left(x_{i}\right)\right)-\left(b_{s, t_{1}}^{\eta}\right)^{\prime}\left(h_{t}\left(x_{i+1}\right)\right) \alpha_{t}\left(h_{t}\left(x_{i+1}\right)\right)\right|^{p} d t \\
& \quad \leq\left|t_{2}-t_{1}\right|\left(\sup _{x \in(-\infty, c)}\left|\left(b_{s, t_{1}}^{\eta}\right)^{\prime}(x)\right| \sup _{t}\left\|\alpha_{t} \circ h_{t}\right\|_{B V_{p}}+\sup _{x, t}\left|\alpha_{t} \|\left(\left.b_{s, t_{1}}^{\eta}\right|_{(-\infty, c)}\right)^{\prime}\right| 1 / p\right) \\
& \quad \leq \bar{C} \frac{\left|t_{2}-t_{1}\right|}{\eta^{1+1 / p-\beta}} .
\end{aligned}
$$

(We used (24) in the last inequality.) The same bounds hold for $c \leq x_{0}<x_{1}<\cdots<x_{N}$, and it is easy to estimate the jump of $b_{s, t_{1}}^{\eta} \circ h_{t_{2}}-b_{s, t_{1}}^{\eta} \circ h_{t_{1}}$ at $x=c$ uniformly in $s$ and $t_{1}, t_{2}$.

We next analyse the contribution of $b_{s, t_{1}}-b_{s, t_{1}}^{\eta}$ to the second term of (30). For this, observe that if $h$ is an orientation-preserving homeomorphism fixing $c$ and $b$ is $\beta$-Hölder on $[-\infty, c]$ and $[c, \infty]$, then $\|b \circ h\|_{B V_{p}} \leq\left.|b|_{(-\infty, c)}\right|_{\beta}+\left.|b|_{[c, \infty)}\right|_{\beta}+\left|b\left(c_{+}\right)-b\left(c_{-}\right)\right|$. 
Then, the last bound of (25) and its analogue for the $\eta$-regularization of $\psi^{\prime}, f_{t}^{\prime \prime}$ and $\partial_{t} f_{t}^{\prime}$ give a constant $C^{\prime}$ so that for all $\left|t_{1}\right|<\epsilon_{p},\left|t_{2}\right|<\epsilon_{p}$ and $\eta \in(0,1)$

$$
\begin{aligned}
\sup _{s \in K}\left\|\left(b_{s, t_{1}}-b_{s, t_{1}}^{\eta}\right) \circ h_{t_{2}}-\left(b_{s, t_{1}}-b_{s, t_{1}}^{\eta}\right) \circ h_{t_{1}}\right\|_{B V_{p}} & \leq 2 \sup _{s, t}\left\|\left(b_{s, t_{1}}-b_{s, t_{1}}^{\eta}\right) \circ h_{t}\right\|_{B V_{p}} \\
& \leq C^{\prime} \eta^{\beta-1 / p} .
\end{aligned}
$$

Taking $\xi \in(0,1)$ and setting $\eta=\left(t_{2}-t_{1}\right)^{\xi /(1+1 / p-\beta)}$, we obtain from (31-32) that $\lim _{\tilde{t} \rightarrow t_{1}} \sup _{t_{2} \in\left[t_{1}, \tilde{t}\right]} \sup _{s \in K}\left\|b_{s, t_{1}} \circ h_{t_{2}}-b_{s, t_{1}} \circ h_{t_{1}}\right\|_{B V_{p}}=0$.

To analyse the first term of (30), we start by noting that since $t \mapsto \partial_{t} h_{t}$ is Lipschitz, there exists a set $\mathcal{D}_{p} \subset\left(-\epsilon_{p}, \epsilon_{p}\right)$ of full Lebesgue measure so that $\partial_{t} h_{t}$ is differentiable at all $t$ in $\mathcal{D}_{p}$. Differentiating twice $f_{t} \circ h_{t}(x)=h_{t} \circ f(x)$ with respect to $t$ and $\dagger$ setting $\alpha_{t}^{2}=\partial_{t t}^{2} h_{t} \circ h_{t}^{-1}$, we obtain for all $x \neq c$ and all $t \in \mathcal{D}_{p}$ that

$$
f_{t}^{\prime \prime}(x) \alpha_{t}(x)^{2}+2 \partial_{t} f_{t}^{\prime}(x) \alpha_{t}(x)+\partial_{t t} f_{t}(x)=\alpha_{t}^{2}\left(f_{t}(x)\right)-f_{t}^{\prime}(x) \alpha_{t}^{2}(x) .
$$

The left-hand side of the above TCE is $\beta$-Hölder in $[-1, c]$ and $[c, 1]$ and continuous in $I$, since $\alpha_{t}(c)=0$ for every small $t$, so it is $\beta$-Hölder continuous. Therefore, by Proposition 2.3, there exist $\epsilon_{p}>0$ and a constant $C^{\prime \prime}$ so that

$$
\left|\alpha_{t}^{2}\right|_{\beta} \leq C^{\prime \prime} \quad \text { for all } t \in \mathcal{D}_{p} .
$$

The fundamental theorem of calculus holds for the Lipschitz (and, therefore, almost everywhere differentiable) function $t \mapsto b_{s, t}$ and gives

$$
\left(b_{s, t_{2}}-b_{s, t_{1}}\right) h_{t_{2}}(x)=\int_{t_{1}}^{t_{2}} \partial_{t} b_{s, t}\left(h_{t_{2}}(x)\right) d t \quad \text { for all } x \neq c .
$$

The first term of (30) may then be estimated via the Hölder inequality and the fundamental theorem of calculus (35), as in (31), but exploiting (34) instead of using $\eta$-regularization. Details are left to the reader.

Finally, to show (28), start from

$$
g_{s, \tilde{t}}(x)-g_{s, t_{1}}(x)-\left(\tilde{t}-t_{1}\right) b_{s, t_{1}}\left(h_{t_{1}}(x)\right)=\int_{t_{1}}^{\tilde{t}}\left(b_{s, t_{2}}\left(h_{t_{2}}(x)\right)-b_{s, t_{1}}\left(h_{t_{1}}(x)\right)\right) d t_{2},
$$

for all $x \neq c$, and use the Hölder inequality and (29) (details are left to the reader).

The analysis of the maps $t \mapsto\left(\psi \circ h_{t}\right)^{k} g_{s, t} / k$ ! for $k \geq 1$ goes along exactly the same lines.

For the higher regularity statement in Theorem 3.1, we use the following result (again, analyticity in $s$ holds).

Lemma 3.4. Let $j \geq 2$. Let $f_{t}$ be a $C^{j+1}$ family of piecewise expanding $C^{j+2}$ unimodal maps in the topological class of $f_{0}$. For any $p>1$ there exists $\epsilon_{p}>0$ so that for any $\psi: I \rightarrow \mathbb{R}$ which is $C^{j+\mathrm{Lip}}$, the map $s \mapsto g_{s, t}$ defined by (16) is $C^{1}$ from $\mathbb{R}$ to the space of $C^{j}$ maps from $\left\{|t|<\epsilon_{p}\right\}$ to $B V_{p}$, and, recalling (1), $\left.\partial_{s} g_{s, t}\right|_{s=u}=\left(\psi \circ h_{t}\right) g_{u, t}$.

$\dagger$ This is similar to the proof of [4, Proposition 2.4], but we make a more careful analysis of what was called $F_{i}$ there. 
Proof. Since the family $f_{t}$ is $C^{j+1}$, the set $\left\{t \mapsto h_{t}(x), x \in I\right\}$ is bounded in $C^{j+\text { Lip }}$ by [4, Proposition 2.4]. Let $\beta \in(1 / p, 1)$ (with $\beta<1 / \sqrt{p}$, say). Assume first $j=2$. Then, by (33), the function $\alpha_{t}^{2}=\partial_{t t}^{2} h_{t} \circ h_{t}^{-1}$, is well defined for all $|t|<\epsilon_{p}$ and there exists $C$ so that $\left|\alpha_{t}^{2}\right|_{\beta} \leq C$ for every $|t|<\epsilon_{p}$. For $j \geq 3$, a higher-order TCE similar to (33) gives that $\alpha_{t}^{j}=\partial_{t^{j}}^{j} h_{t} \circ h_{t}^{-1}$ is $\beta$-Hölder for all $|t|<\epsilon_{p}$. We put $\alpha_{t}^{1}=\alpha_{t}$.

Then, computing $\partial_{t^{j}}^{j} g_{s, t}(x)$ at $x \neq c$ gives $b_{s, t}^{(j)}\left(h_{t}(x)\right)$, where $b_{s, t}^{(j)}$ is an expression involving derivatives of order at most $j$ of $\psi(x)$, functions $\alpha_{t}^{\ell}$, for $1 \leq \ell \leq j$, and derivatives (in $x, t$, or mixed) of total order at most $j$ of $f_{t}^{\prime}(x)$, in the numerator, and $\left|f_{t}^{\prime}(x)\right|^{m}$ for $m \geq 1$ in the denominator. Our differentiability assumptions on $\psi$ and the family $f_{t}$ then allow us to proceed as in the proof of Lemma 3.3 (using Taylor series of higher order).

\section{Recovering the linear response formula}

Here we give a slightly different proof of the differentiability of $R(t)=\int \psi d \mu_{t}$, where $\mu_{t}$ is the SRB measure of $f_{t}$, still relying heavily on Proposition 2.3 (via Lemma 4.2). The advantage with respect to Theorem 3.1 is that we recover the formula for $\left.\partial_{t} R(t)\right|_{t=0}$, and we need only assume that $\psi$ is $C^{0}$. (In particular, this gives a new proof of [4, Theorem 5.1].) We also obtain new information in Corollary 4.4 by combining Theorems 3.1 and 4.1 .

We need notation. By [1, Proposition 3.3], we may decompose the invariant density of a piecewise expanding $C^{3}$ unimodal mixing map $f_{t}$ as $\rho_{t}=\rho_{\mathrm{reg}, t}+\rho_{\mathrm{sal}, t}$, where $\rho_{\text {reg }, t} \in B V \cap C^{0}, \rho_{\text {reg }, t}^{\prime} \in B V$, and

$$
\rho_{\mathrm{sal}, t}=\sum_{k=1}^{M_{f}} s_{k, t} H_{c_{k, t}} .
$$

(Here, $H_{u}(x)$ denotes the Heaviside function $H_{u}(x)=-1$ if $x<u, H_{u}(x)=0$ if $x>u$ and $H_{u}(u)=-1 / 2$.) If $M_{f}=\infty$, then it is not difficult to show that (see e.g. $[1,4]$, noting that if $c_{1, t}$ is preperiodic but not periodic our notation is slightly different than the notation there)

$$
s_{k, t}=\frac{s_{1, t}}{\left(f^{k-1}\right)^{\prime}\left(c_{1, t}\right)} \quad \text { for all } k \geq 1 .
$$

We simply write $\rho_{0}=\rho=\rho_{\text {reg }}+\rho_{\text {sal }}$.

To compute the formula for the derivative, we assume, as in [4], that $v=\left.\partial_{t} f_{t}\right|_{t=0}$ is of the form $v=X \circ f_{0}$ for a $C^{2}$ function $\dagger X: I \rightarrow \mathbb{R}$.

THEOREM 4.1. Let $f_{t}$ be a $C^{2}$ family of piecewise expanding $C^{3}$ unimodal maps. Assume that $f_{0}$ is good and mixing, that $f_{t}$ is tangent to the topological class of $f_{0}$, and that $v=\left.\partial_{t} f_{t}\right|_{t=0}=X \circ f_{0}$ for a $C^{2}$ function $X$. Then, as Radon measures,

$$
\lim _{t \rightarrow 0} \frac{\mu_{t}-\mu_{0}}{t}=-\alpha \rho_{\text {sal }}^{\prime}-\left(\mathrm{id}-\mathcal{L}_{0}\right)^{-1}\left(X^{\prime} \rho_{\text {sal }}+\left(X \rho_{\text {reg }}\right)^{\prime}\right) d x,
$$

where the function $\alpha$ is given by (4), and the operator $\mathcal{L}_{0}=\widetilde{\mathcal{L}}_{0,0}$ is defined by (17). In addition, $\alpha$ is $\beta$-Hölder for any $\beta<1$.

$\dagger$ See also the beginning of $[\mathbf{2 1}, \S 17]$. 
Proof. Set $f=f_{0}$ for convenience. By Proposition A.1, we can assume that $f_{t}$ lies in the topological class of $f$, denoting the conjugacies by $h_{t}$ as usual. In the beginning of the proof of Theorem 3.1, we observed that the transfer operator $\widetilde{\mathcal{L}}_{t}=\widetilde{\mathcal{L}}_{0, t}$ on $B V_{p}$ is conjugated to the transfer operator $\mathcal{L}_{t}$ on $B V_{p}$, and that there exists $p_{0}$ so that for any $p \in\left[1, p_{0}\right)$ there is $\epsilon_{p}>0$ so that for each $|t|<\epsilon_{p}$ the operator $\widetilde{\mathcal{L}}_{t}$ acting on $B V_{p}$ has a maximal eigenvalue equal to one which is simple, and the rest of the spectrum lies in a disc of strictly smaller radius. The fixed points of $\widetilde{\mathcal{L}}_{t}$ and its dual, $\widetilde{\rho}_{t}=\rho_{t} \circ h_{t}$ and $v_{t}$ from (19), were also introduced in the proof of Theorem 3.1.

From now on, we fix $p \in\left(1, p_{0}\right)$.

We next show that $t \mapsto \tilde{\rho}(t) \in B V_{p}$ and $t \mapsto v_{t} \in B V_{p}^{*}$ are differentiable at $t=0$. By [4, Proposition 2.4 and Corollary 2.6] $v$ is horizontal for $f_{0}, t \mapsto h_{t}(x)$ is differentiable, uniformly in $x \in I$, and $\alpha=\left.\partial_{t} h_{t}\right|_{t=0}$ is continuous, with $\alpha(c)=0, \alpha\left(c_{1}\right)=X(c)$, and $\alpha$ is the unique bounded solution (4) to the TCE (3). In addition, Proposition 2.3 gives that $\alpha$ is $\beta$-Hölder for arbitrary $\beta<1$ (we take $\beta \in(1 / p, 1 / \sqrt{p})$ ).

Our assumptions on $f_{t}$ then imply that $v^{\prime}$ is $C^{1}$ and the following operator is bounded on $B V_{p}$ :

$$
\mathcal{M} \varphi(x)=-\sum_{f(y)=x} \frac{f^{\prime \prime}(y) \alpha(y)+v^{\prime}(y)}{\left|f^{\prime}(y)\right| f^{\prime}(y)} \varphi(y) .
$$

(Write $\mathcal{M}$ as $\mathcal{L}_{0}$ composed with a multiplication operator, as in (20), and use (14).) Lemma 4.2 below easily implies that $t \mapsto \tilde{\mathcal{L}}_{t}$ is differentiable as an operator on $B V_{p}$, and that

$$
\left.\partial_{t} \tilde{\mathcal{L}}_{t}\right|_{t=0}=\mathcal{M}
$$

As in the proof of Theorem 3.1, perturbation theory then gives that $t \mapsto \tilde{\rho}_{t} \in B V_{p}$ and $t \mapsto v_{t} \in B V_{p}^{*}$ are differentiable at $t=0$. In particular, since $\tilde{\rho}_{0}=\rho_{0}$,

$$
\lim _{t \rightarrow 0}\left\|\tilde{\rho}_{t}-\rho_{0}\right\|_{B V_{p}}=0 \text {. }
$$

We next show that $t \mapsto \mu_{t}=\rho_{t} d x$ is differentiable as a Radon measure, exploiting the formula for $\mathcal{M}$ to obtain the claimed formula for $\left.\partial_{t} \mu_{t}\right|_{t=0}$. Fix $\psi: I \rightarrow \mathbb{C}$ continuous. Since $\tilde{\rho}_{0}=\rho_{0}$, we can decompose

$$
\int \psi \rho_{t} d x-\int \psi \rho_{0} d x=\int \psi \rho_{t} d x-\int \psi \tilde{\rho}_{t} d x+\int \psi \tilde{\rho}_{t} d x-\int \psi \tilde{\rho}_{0} d x .
$$

We now see that

$$
\lim _{t \rightarrow 0} \frac{\int \psi\left(\rho_{t}-\rho_{t} \circ h_{t}\right) d x}{t}=-\int \psi \alpha \rho_{0}^{\prime} .
$$

In view of (42), note first that $s_{k, t} \rightarrow s_{k}$ as $t \rightarrow 0$ : Indeed, (40) gives (in $B V_{p}$ )

$$
\lim _{t \rightarrow 0} \tilde{\rho}_{t}=\lim _{t \rightarrow 0}\left(\rho_{\text {reg }, t} \circ h_{t}+\sum_{k=1}^{M_{f}} s_{k, t} H_{c_{k}}\right)=\tilde{\rho}_{0}=\rho_{\text {reg }}+\sum_{k=1}^{M_{f}} s_{k} H_{c_{k}} .
$$

(We gave another proof of $\lim _{t \rightarrow 0} s_{k, t}=s_{k}$ in Step 1 of [4, Proof of Theorem 5.1].)

Decompose $\rho_{t}-\rho_{t} \circ h_{t}$ in (42) into $\rho_{\mathrm{sal}, t}-\rho_{\mathrm{sal}, t} \circ h_{t}+\rho_{\text {reg, } t}-\rho_{\mathrm{reg}, t} \circ h_{t}$. For the singular term, we have in the sense of Radon measures:

$$
\lim _{t \rightarrow 0} \frac{\rho_{\mathrm{sal}, t}-\rho_{\mathrm{sal}, t} \circ h_{t}}{t}=-\sum_{k=1}^{M_{f}} \alpha\left(c_{k}\right) s_{k} \operatorname{Dirac}_{c_{k}}=-\alpha \rho_{\mathrm{sal}}^{\prime} .
$$


(Just use that $s_{k, t} \rightarrow s_{k}$ and (36), which implies that the $s_{k, t}$ decay exponentially in $k$ uniformly in $t$.)

We claim that the contribution of the regular term $\rho_{\text {reg, } t}$ in the decomposition of (42) is $-\int \psi \alpha \rho_{\text {reg }}^{\prime} d x$. In view of this, we first note that if $x \in\left[-1, c_{1}\right)$ is not along the postcritical orbit, we find, using $\left(\rho_{\mathrm{reg}, t}\right)^{\prime}(y)=\left(\rho_{t}\right)^{\prime}(y)$ if $y$ is not on the postcritical orbit, that

$$
\left(\rho_{\text {reg }, t}\right)^{\prime}(x)=\left(\rho_{t}\right)^{\prime}(x)=\left(\mathcal{L}_{t}\left(\rho_{t}\right)\right)^{\prime}(x)=\sum_{f_{t}(y)=x} \frac{\left(\rho_{\text {reg }, t}\right)^{\prime}(y)}{\left|f_{t}^{\prime}(y)\right| f_{t}^{\prime}(y)}-\frac{\rho_{t}(y) f_{t}^{\prime \prime}(y)}{\left|f_{t}^{\prime}(y)\right|\left(f_{t}^{\prime}(y)\right)^{2}} .
$$

Next, by [1, Proposition 3.3], $\rho_{\text {reg, } t}^{\prime} \in B V$. The proof of [1, Proposition 3.3] implies that the discontinuities of $\rho_{\text {reg, } t}^{\prime}$ lie in the set $\left\{c_{k, t}\right\}$. In other words, we may decompose

$$
\rho_{\text {reg }, t}=\rho_{\text {regreg }, t}+\rho_{\text {regsal }, t},
$$

with $\rho_{\text {regreg }, t}^{\prime}=\left(\rho_{\text {reg, }, t}^{\prime}\right)_{\text {reg }}$ continuous (that is, $\rho_{\text {regreg }, t}$ is $C^{1}$ ), and

$$
\rho_{\text {regsal }, t}^{\prime}=\left(\rho_{\text {reg }, t}^{\prime}\right)_{\text {sal }}=\sum_{k=1}^{M_{f}} s_{k, t}^{\prime} H_{c_{k, t}} .
$$

By the proof of [1, Proposition 3.3] $\rho_{\text {regreg, } t}$ is $C^{1}$ uniformly in $t$. We next show that the $s_{k, t}^{\prime}$ decay exponentially uniformly in $t$. For this, introduce the notation $\dagger$

$$
\begin{aligned}
E_{1, t}:= & \left(-\frac{\rho_{\mathrm{reg}, t}(c) f_{t}^{\prime \prime}\left(c_{-}\right)}{\left(f_{t}^{\prime}\left(c_{-}\right)\right)^{3}}+\frac{\rho_{\mathrm{reg}, t}(c) f_{t}^{\prime \prime}\left(c_{+}\right)}{\left(f_{t}^{\prime}\left(c_{+}\right)\right)^{3}}\right) \\
& +\sum_{k \geq 2, c_{k-1, t}>c} s_{k-1, t}\left(\frac{f_{t}^{\prime \prime}\left(c_{-}\right)}{\left(f_{t}^{\prime}\left(c_{-}\right)\right)^{3}}-\frac{f_{t}^{\prime \prime}\left(c_{+}\right)}{\left(f_{t}^{\prime}\left(c_{+}\right)\right)^{3}}\right) \\
E_{k, t}:= & \frac{s_{k-1, t} f_{t}^{\prime \prime}\left(c_{k-1, t}\right)}{\left(f_{t}^{\prime}\left(c_{k-1, t}\right)\right)^{3}}, \quad k \geq 2, \\
E_{1, t}^{\prime}:= & -\frac{\left(\rho_{\mathrm{reg}, t}\right)^{\prime}(c)}{\left(f_{t}^{\prime}\left(c_{-}\right)\right)^{2}}+\frac{\left(\rho_{\mathrm{reg}, t}\right)^{\prime}(c)}{\left(f_{t}^{\prime}\left(c_{+}\right)\right)^{2}} \\
E_{k, t}^{\prime}:= & \frac{s_{k-1, t}^{\prime}}{\left(f_{t}^{\prime}\left(c_{k-1, t}\right)\right)^{2}}, \quad k \geq 2 .
\end{aligned}
$$

Then equating the jump at $c_{k, t}$ in both sides of (45) implies that

$$
s_{k, t}^{\prime}=E_{k, t}^{\prime}-E_{k, t},
$$

and thus uniform exponential decay of the $s_{k, t}^{\prime}$, which are determined by $s_{1, t}^{\prime}, s_{1, t}$, and the postcritical first and second derivatives.

The argument above giving $s_{k, t} \rightarrow s_{k}$ also yields $s_{k, t}^{\prime} \rightarrow s_{k}^{\prime}$ (just differentiate once). Therefore, just like in (44), we have

$$
\begin{aligned}
\lim _{t \rightarrow 0} \int \psi \frac{\rho_{\text {regsal }, t}-\rho_{\text {regsal }, t} \circ h_{t}}{t} d x & =-\sum_{k=1}^{M_{f}} \int \alpha(x) s_{k}^{\prime} \psi(x) H_{c_{k}}(x) d x \\
& =\int \psi \alpha \rho_{\text {regsal }}^{\prime} d x .
\end{aligned}
$$

$\dagger$ If $c$ is periodic, then $\left(\rho_{\text {reg, }, t}\right)^{\prime}(c)$ may be undefined, but $\left(\rho_{\text {reg, }, t}\right)^{\prime}\left(c_{ \pm}\right)$are both defined. 
In view of handling the term $\rho_{\text {regreg, } t}$ from (46), observe that

$$
\lim _{t \rightarrow 0}\left\|\varphi-\varphi \circ h_{t}\right\|_{B V}=0 \text { for all } \varphi \in C^{1} .
$$

Indeed, for $\delta>0$ and any partition $x_{0}<\cdots<x_{i}<x_{i+1}<\cdots<x_{n}$ let $N \leq n$ be so that $\min \left(x_{N}, \inf _{t} h_{t}\left(x_{N}\right)\right)>1-\delta$, and since $\left|h_{t}(y)-y\right|=O(t)$ uniformly in $y$, take $t_{0}$ so that $\left|x_{i}-h_{t}\left(x_{i}\right)\right|<\delta / N$ for all $i \leq N$ and $|t|<t_{0}$. Then use

$$
\begin{aligned}
& \sum_{i=0}^{n-1}\left|\varphi\left(x_{i}\right)-\varphi\left(x_{i+1}\right)-\varphi\left(h_{t}\left(x_{i}\right)\right)+\varphi\left(h_{t}\left(x_{i+1}\right)\right)\right| \\
& \leq 2 \sum_{i=0}^{N}\left|\varphi\left(x_{i}\right)-\varphi\left(h_{t}\left(x_{i}\right)\right)\right|+\sum_{i=N+1}^{n-1}\left|\varphi\left(x_{i}\right)-\varphi\left(x_{i+1}\right)\right| \\
& \quad+\sum_{i=N+1}^{n-1}\left|\varphi\left(h_{t}\left(x_{i}\right)\right)-\varphi\left(h_{t}\left(x_{i+1}\right)\right)\right| .
\end{aligned}
$$

Since the $C^{1}$ norm of $\rho_{\text {regreg, } t}$ is bounded uniformly in $t$, (48) and (40) together with $s_{k, t}^{\prime} \rightarrow s_{k}^{\prime}$ easily imply that

$$
\begin{aligned}
& \lim _{t \rightarrow 0}\left\|\rho_{\text {regreg }, t}-\rho_{\text {regreg }}\right\|_{B V_{p}} \\
& \quad=\lim _{t \rightarrow 0}\left\|\rho_{\text {regreg, } t}-\rho_{\text {regreg }, t} \circ h_{t}+\rho_{\text {regreg }, t} \circ h_{t}-\rho_{\text {regreg }}\right\|_{B V_{p}}=0 .
\end{aligned}
$$

(Note for the record that, since $s_{k, t}^{\prime} \rightarrow s_{k}^{\prime}$, with $t$-uniformly $k$-exponentially decaying $s_{k, t}^{\prime}$, this implies $\lim _{t \rightarrow 0}\left\|\rho_{\text {reg, } t}-\rho_{\text {reg }}\right\|_{B V_{p}}=0$.) Then, by the mean value theorem and the $x$-uniform differentiability of $t \mapsto h_{t}(x)$

$$
\begin{aligned}
\lim _{t \rightarrow 0} \int \psi \frac{\rho_{\text {regreg }, t}-\rho_{\text {regreg }, t} \circ h_{t}}{t} d x \\
\quad=\lim _{t \rightarrow 0} \int \psi(x) \frac{\rho_{\text {regreg }, t}(x)-\rho_{\text {regreg }, t}\left(h_{t}(x)\right)}{x-h_{t}(x)} \frac{x-h_{t}(x)}{t} d x \\
=\lim _{t \rightarrow 0} \int \psi(x) \rho_{\text {regreg }, t}^{\prime}\left(x_{t}\right) \frac{x-h_{t}(x)}{t} d x \\
=-\lim _{t \rightarrow 0} \int \psi(x) \rho_{\text {regreg }, t}^{\prime}\left(x_{t}\right) \alpha(x) d x \\
=-\int \psi(x) \rho_{\text {regreg }, 0}^{\prime}(x) \alpha(x) d x,
\end{aligned}
$$

where $x_{t}$ is in the interval between $x$ and $h_{t}(x)$, and we used in the last line that $\rho_{\text {regreg, } t}^{\prime}$ is continuous on the compact interval $I$, uniformly in $t$, together with (49), Proposition 2.3, and (13). Putting (44), (47) and (50) together, we find (42).

We now turn to the estimation of the term $\left(\int \psi \tilde{\rho}_{t} d x-\int \psi \tilde{\rho}_{0} d x\right) / t$ from (41). In view of this, note that (39) implies that (as operators on $B V_{p}$ )

$$
\left.\partial_{t}\left(z-\tilde{\mathcal{L}}_{t}\right)^{-1}\right|_{t=0}=\left(z-\mathcal{L}_{0}\right)^{-1} \mathcal{M}\left(z-\mathcal{L}_{0}\right)^{-1} .
$$

Therefore, writing the spectral projectors as Cauchy integrals, we obtain by a simple residue computation, since $\left(z-\mathcal{L}_{0}\right)^{-1} \rho_{0}=\rho_{0} /(z-1)$, that

$$
\left.\partial_{t}\left(v_{t}\left(\rho_{0}\right) \tilde{\rho}_{t}\right)\right|_{t=0}=\left(\mathrm{id}-\mathcal{L}_{0}\right)^{-1}\left(\mathrm{id}-\Pi_{0}\right) \mathcal{M} \rho_{0},
$$

where $\Pi_{0}(\varphi)=\rho_{0} \int \varphi d x$. 
Next, we claim that we have (in $B V_{p}$ )

$$
-\alpha \rho_{\text {reg }}^{\prime}+\left(\mathrm{id}-\mathcal{L}_{0}\right)^{-1}\left(\mathrm{id}-\Pi_{0}\right)\left(\mathcal{M} \rho_{0}\right)=-\left(\mathrm{id}-\mathcal{L}_{0}\right)^{-1}\left(\mathrm{id}-\Pi_{0}\right)\left(X^{\prime} \rho_{0}+X \rho_{\text {reg }}^{\prime}\right) .
$$

(Recall from [1, Proof of Proposition 4.4] that $\Pi_{0}\left(X^{\prime} \rho_{0}+X \rho_{\text {reg }}^{\prime}\right)=0$.) Since the TCE (3) implies, using $v^{\prime}=\left(X^{\prime} \circ f\right) \cdot f^{\prime}$, that

$$
\mathcal{M} \rho_{0}(x)=(X(x)-\alpha(x)) \sum_{f(y)=x} \frac{f^{\prime \prime}(y)}{\left|f^{\prime}(y)\right| f^{\prime}(y)^{2}} \rho_{0}(y)-X^{\prime}(x) \rho_{0}(x),
$$

to prove (10), it suffices to show

$$
-\alpha \rho_{\text {reg }}^{\prime}+\left(\mathrm{id}-\mathcal{L}_{0}\right)^{-1}\left(\mathrm{id}-\Pi_{0}\right)(X-\alpha)\left(\widetilde{\mathcal{M}} \rho_{0}\right)=-\left(\mathrm{id}-\mathcal{L}_{0}\right)^{-1}\left(\mathrm{id}-\Pi_{0}\right)\left(X \rho_{\text {reg }}^{\prime}\right),
$$

where $\widetilde{\mathcal{M}} \varphi(x)=\sum_{f(y)=x}\left(f^{\prime \prime}(y) /\left(\left|f^{\prime}(y)\right| f^{\prime}(y)^{2}\right)\right) \varphi(y)$. It follows from (45) that for any $x \in I$ which is not on the postcritical orbit

$$
\widetilde{\mathcal{M}}\left(\rho_{0}\right)(x)=\sum_{f(y)=x} \frac{\rho_{\mathrm{reg}}^{\prime}(y)}{\left|f^{\prime}(y)\right| f^{\prime}(y)}-\rho_{\mathrm{reg}}^{\prime}(x) .
$$

In other words, we have (in $B V_{p}$ )

$$
\widetilde{\mathcal{M}}\left(\rho_{0}\right)=\overline{\mathcal{M}}\left(\rho_{\text {reg }}^{\prime}\right)-\rho_{\text {reg }}^{\prime},
$$

where $\overline{\mathcal{M}} \varphi(x)=\sum_{f(y)=x}\left(\varphi(y) /\left(\left|f^{\prime}(y)\right| f^{\prime}(y)\right)\right)$. So we have reduced the claim (52) to

$$
-\alpha \rho_{\text {reg }}^{\prime}-\left(\mathrm{id}-\mathcal{L}_{0}\right)^{-1}\left(\mathrm{id}-\Pi_{0}\right)\left(\mathcal{L}_{0}-\mathrm{id}\right)\left(\alpha \rho_{\text {reg }}^{\prime}\right)=0,
$$

that is, using $\Pi_{0} \mathcal{L}_{0}=\Pi_{0}$,

$$
\left(\mathcal{L}_{0}-\mathrm{id}\right)\left(\alpha \rho_{\text {reg }}^{\prime}\right)=\left(\mathrm{id}-\Pi_{0}\right)\left(\mathcal{L}_{0}-\mathrm{id}\right)\left(\alpha \rho_{\text {reg }}^{\prime}\right)=\left(\mathcal{L}_{0}-\mathrm{id}\right)\left(\alpha \rho_{\text {reg }}^{\prime}\right) .
$$

Finally, since $\rho_{0} \in B V_{p}$, and since $t \mapsto v_{t}$ and $t \mapsto \tilde{\rho}_{t}$ are differentiable in $B V_{p}$ and $B V_{p}^{*}$, respectively, we have (in $B V_{p}$ )

$$
\left.\partial_{t}\left(v_{t}\left(\rho_{0}\right) \tilde{\rho}_{t}\right)\right|_{t=0}=\left.\partial_{t}\left(v_{t}\left(\rho_{0}\right)\right)\right|_{t=0} \rho_{0}+\left.\partial_{t}\left(\tilde{\rho}_{t}\right)\right|_{t=0} .
$$

Take the Lebesgue average of both sides of (53). Since $\partial_{t} \int \tilde{\rho}_{t} d x=0$ (because each $\tilde{\rho}_{t} d t$ is a probability), and since

$$
-\int\left(\mathrm{id}-\mathcal{L}_{0}\right)^{-1}\left(X^{\prime} \rho_{\text {sal }}+\left(X \rho_{\text {reg }}\right)^{\prime}\right) d x=0
$$

(again use $\left.\Pi_{0}\left(X^{\prime} \rho_{0}+X \rho_{\text {reg }}^{\prime}\right)=0\right)$, we find that $\left.\partial_{t}\left(v_{t}\left(\rho_{0}\right)\right)\right|_{t=0} \int \rho_{0} d x=0$. Therefore, $\left.\partial_{t}\left(v_{t}\left(\rho_{0}\right)\right)\right|_{t=0}$, and putting together (41), (42), (51), (52) and (53), we have proved the theorem.

We have (a simplification of Lemma 3.3) the following result.

LEMMA 4.2. Let $f_{t}$ be a $C^{2}$ family of piecewise expanding $C^{3}$ unimodal maps in the topological class of $f_{0}$. Set $v=\left.\partial_{t} f_{t}\right|_{t=0}$. For any $p>1$ the map $t \mapsto g_{t}=1 /\left|f_{t}^{\prime} \circ h_{t}\right| \in$ $B V_{p}$ is $C^{1}$ in a neighbourhood of 0 , and $\left.\partial_{t} g_{t}\right|_{t=0}=-\left(f_{0}^{\prime \prime} \alpha+v^{\prime}\right) /\left(\left|f_{0}^{\prime}\right| f_{0}^{\prime}\right)$.

Proof. Differentiability follows from Lemma 3.3 applied to $\psi \equiv 0$. The value of the derivative is given by (26) and (27) in the proof of that lemma, since $\left.\partial_{t} f_{t}^{\prime}\right|_{t=0}=v^{\prime}$. 
Remark 4.3. We have the following strengthening of Lemma 4.2 if $f_{t}$ is a $C^{3}$ family of piecewise expanding $C^{4}$ unimodal maps: for any $p>1$ the map $t \mapsto g_{t}=1 /\left|f_{t}^{\prime} \circ h_{t}\right|$ $\in B V_{p}$ is $C^{2}$ in a neighbourhood of 0 , and $\left|g_{t}-g_{0}+t\left(\left(f_{0}^{\prime \prime} \alpha+v^{\prime}\right) /\left(\left|f_{0}^{\prime}\right| f_{0}^{\prime}\right)\right)\right|=O(t)$. Recalling (38)-(39), this implies that $\left\|\left(\tilde{\mathcal{L}}_{t}-\mathcal{L}_{0}\right) / t-\mathcal{M}\right\|_{B V_{p}}=O(t)$.

We obtain the following (new) result as a corollary of Theorems 3.1 and 4.1.

COROLlaRY 4.4. If $f_{t}$ is a $C^{2}$ family of piecewise expanding $C^{3}$ unimodal maps in the topological class of $f_{0}$, and if $\left.\partial_{t} f_{t}\right|_{t=0}=X \circ f_{0}$ for a $C^{2}$ function $X$, then there exists $\epsilon>0$ so that $t \mapsto \mu_{t}$ is $C^{1}$ from $(-\epsilon, \epsilon)$ to Radon measures.

In particular, under the assumptions of Corollary 4.4, the Radon measure

$$
-\alpha_{t} \rho_{\mathrm{sal}, t}^{\prime}-\left(\mathrm{id}-\mathcal{L}_{t}\right)^{-1}\left(X_{t}^{\prime} \rho_{\mathrm{sal}, t}+\left(X_{t} \rho_{\mathrm{reg}, t}\right)^{\prime}\right) d x
$$

(recall (37)) is continuous as a function of $t$. (Here, $\alpha_{t}$ solves (3) for $f_{t}$ and $v_{t}=\left.\partial_{s} f_{s}\right|_{s=t}$, and $X_{t} \circ f_{t}=v_{t}$.) This fact is not clear a priori from the formula.

Remark 4.5. We expect that a careful analysis of the term (42) for $C^{1}$ functions $\psi$ would allow us to bypass the reference to Theorem 3.1 in the proof of Corollary 4.4.

Proof of Corollary 4.4. We want to show that $\left.t \mapsto \partial_{u} \mu_{u}\right|_{u=t}=\tilde{\mu}_{t}$ is continuous: we know that $\tilde{\mu}_{t}$ exists for all small $t$ (as a Radon measure) by Theorem 4.1. Clearly, $\left|\int \psi d \tilde{\mu}_{t}\right| \leq C \sup |\psi|$ for all continuous $\psi$ and all small enough $t$.

Assume for a contradiction that $t \mapsto \tilde{\mu}_{t}$ is discontinuous at $t_{0}$. This means that there exist $\psi \in C^{0}$, with $\sup |\psi|=1, \delta>0$, and a sequence $t_{m}$ with $\left|t_{m}-t_{0}\right|<1 / m$, so that $\left|\int \psi d \tilde{\mu}_{t_{0}}-\int \psi d \tilde{\mu}_{t_{m}}\right|>\delta$ for all $m$. Take $\tilde{\psi} \in C^{1}$ so that $\sup |\psi-\tilde{\psi}|<\delta / 4$. Then $\left|\int \tilde{\psi} d \tilde{\mu}_{t_{0}}-\int \tilde{\psi} d \tilde{\mu}_{t_{m}}\right|>\delta / 2$ for all $m$. However, Theorem 3.1 implies $\mid \int \tilde{\psi} d \tilde{\mu}_{t_{0}}-$ $\int \tilde{\psi} d \tilde{\mu}_{t_{m}} \mid<\delta$ if $m$ is large enough, a contradiction.

\section{A. Appendix. A consequence of the Keller-Liverani bounds from [4]}

We state here for the record an immediate corollary of [4, Proposition 3.3] which was based on results in [16] (see Remark 2.1 and note that the assumptions below imply $\left.\sup _{I}\left|f_{t}-\tilde{f}_{t}\right|=O\left(t^{2}\right)\right)$.

Proposition A.1. Let $f_{t}$ be a $C^{2}$ family of piecewise expanding $C^{2}$ unimodal maps. Assume that $f_{0}$ is mixing and good, and that $f_{t}$ is tangent to the topological class of $f_{0}$, denoting by $\tilde{f}_{t}$ a family in the topological class of $f_{0}$ with $\tilde{f}_{0}=f_{0}$ and $\left.\partial f_{t}\right|_{t=0}=\left.\partial \tilde{f}_{t}\right|_{t=0}$.

Let $\mu_{t}=\rho_{t} d x$ and $\tilde{\mu}_{t}=\tilde{\rho}_{t} d x$ be the SRB measures of $f_{t}$ and $\tilde{f}_{t}$, respectively. Then for any $\xi<2$ there exists $C>0$ so that for all small $t$

$$
\left\|\rho_{t}-\tilde{\rho}_{t}\right\|_{L^{1}(\text { Leb })} \leq C|t|^{\xi} .
$$

Acknowledgements. V.B. is partially supported by ANR-05-JCJC-0107-01. D.S. is partially supported by CNPq 470957/2006-9 and 310964/2006-7, FAPESP 2003/03107-9. D.S. is grateful to the DMA of École Normale Supérieure for their hospitality during a visit where a crucial part of this work was done. We thank the anonymous referee for comments which helped us improve the presentation. 


\section{REFERENCES}

[1] V. Baladi. On the susceptibility function of piecewise expanding interval maps. Comm. Math. Phys. 275 (2007), 839-859.

[2] V. Baladi. Linear response despite critical points. Nonlinearity 21 (2008), T81-T90.

[3] V. Baladi and A. Hachemi. A local limit theorem with speed of convergence for euclidean algorithms and diophantine costs. Ann. Inst. H. Poincaré Probab. Statist. 44 (2008), 749-770.

[4] V. Baladi and D. Smania. Linear response formula for piecewise expanding unimodal maps. Nonlinearity 21 (2008), 677-711.

[5] V. Baladi and D. Smania. Smooth deformations of piecewise expanding unimodal maps. Discrete Contin. Dyn. Syst. Ser. A 23 (2009), 685-703.

[6] V. Baladi and D. Smania. Analyticity of the SRB measure for holomorphic families of quadratic-like Collet-Eckmann maps. Proc. Amer. Math. Soc. 137 (2009), 1431-1437.

[7] M. Bruneau. Variation Totale d'une Fonction (Lecture Notes in Mathematics, 413). Springer, Berlin, 1974.

[8] J. Buzzi. Private communication (2007).

[9] S. Gouëzel and C. Liverani. Banach spaces adapted to Anosov systems. Ergod. Th. \& Dynam. Sys. 26 (2006), 189-217.

[10] S. Gouëzel and C. Liverani. Compact locally maximal hyperbolic sets for smooth maps: fine statistical properties. J. Differential Geom. 79 (2008), 433-477.

[11] T. Kato. Perturbation Theory for Linear Operators. Springer, Berlin, 1966.

[12] A. Katok, G. Knieper, M. Pollicott and H. Weiss. Differentiability of entropy for Anosov and geodesic flows. Bull. Amer. Math. Soc. 22 (1990), 285-293.

[13] A. Katok, G. Knieper, M. Pollicott and H. Weiss. Differentiability and analyticity of topological entropy for Anosov and geodesic flows. Invent. Math. 98 (1989), 581-597.

[14] G. Keller. Stochastic stability in some chaotic dynamical systems. Monatsh. Math. 94 (1982), 313-333.

[15] G. Keller. Generalized bounded variation and applications to piecewise monotonic transformations. Z. Wahrsch. verw. Gebiete 69 (1985), 461-478.

[16] G. Keller and C. Liverani. Stability of the spectrum for transfer operators. Ann. Sc. Norm. Super. Pisa 28 (1999), 141-152.

[17] G. Keller and T. Nowicki. Spectral theory, zeta functions and the distribution of periodic points for Collet-Eckmann maps. Comm. Math. Phys. 149 (1992), 31-69.

[18] D. Ruelle. Differentiation of SRB states. Comm. Math. Phys. 187 (1997), 227-241; Corrections and complements. Comm. Math. Phys. 234, (2003), 185-190.

[19] D. Ruelle. Nonequilibrium statistical mechanics near equilibrium: computing higher-order terms. Nonlinearity 11 (1998), 5-18.

[20] D. Ruelle. Application of hyperbolic dynamics to physics: some problems and conjectures. Bull. Amer. Math. Soc. 41 (2004), 275-278.

[21] D. Ruelle. Structure and $f$-dependence of the A.C.I.M. for a unimodal map $f$ of Misiurewicz type. Comm. Math. Phys. to appear.

[22] Sh. Wong. Hölder continuous derivatives and ergodic theory. J. London Math. Soc. 22 (1980), 506-520.

[23] L.-S. Young. What are SRB measures, and which dynamical systems have them?. J. Statist. Phys. 108 (2002), 733-754. 